\title{
A fast spectral method for the Boltzmann equation for monatomic gas mixtures
}

\author{
Lei $\mathrm{Wu}^{a 1}$, Jun Zhang ${ }^{a}$, Jason M. Reese ${ }^{b}$, Yonghao Zhang ${ }^{a}$ \\ a James Weir Fluids Laboratory, Department of Mechanical and Aerospace Engineering, University of \\ Strathclyde, Glasgow G1 1XJ, UK \\ ${ }^{b}$ School of Engineering, University of Edinburgh, Edinburgh EH9 3JL, UK
}

\begin{abstract}
Although the fast spectral method has been established for solving the Boltzmann equation for single-species monatomic gases, its extension to gas mixtures is not easy because of the non-unitary mass ratio between the different molecular species. The conventional spectral method can solve the Boltzmann collision operator for binary gas mixtures but with a computational cost of the order $m_{r}^{3} N^{6}$, where $m_{r}$ is the mass ratio of the heavier to the lighter species, and $N$ is the number of frequency nodes in each frequency direction. In this paper, we propose a fast spectral method for binary mixtures of monatomic gases that has a computational cost $O\left(\sqrt{m_{r}} M^{2} N^{4} \log N\right)$, where $M^{2}$ is the number of discrete solid angles. The algorithm is validated by comparing numerical results with analytical BobylevKrook-Wu solutions for the spatially-homogeneous relaxation problem, for $m_{r}$ up to 36 . In spatially-inhomogeneous problems, such as normal shock waves and planar Fourier/Couette flows, our results compare well with those of both the numerical kernel and the direct simulation Monte Carlo methods. As an application, a two-dimensional temperature-driven flow is investigated, for which other numerical methods find it difficult to resolve the flow field at large Knudsen numbers. The fast spectral method is accurate and effective in simulating highly rarefied gas flows, i.e. it captures the discontinuities and fine structures in the velocity distribution functions.
\end{abstract}

Keywords: Boltzmann equation, gas mixtures, Fourier spectral method, rarefied gas dynamics

\section{Introduction}

There are a large number of applications for the Boltzmann equation (BE) in modeling rarefied gas flows frequently encountered in high-altitude aerodynamics, vacuum technologies, and microelectromechanical systems. While there is a great need for efficient and accurate methods for solving the $\mathrm{BE}$, this is a challenge because the $\mathrm{BE}$ employs a molecular velocity distribution function (VDF) defined in a six-dimensional phase space to describe the system state, and the Boltzmann collision operator is highly complicated.

\footnotetext{
${ }^{1}$ Corresponding author. E-mail address: lei.wu.100@strath.ac.uk (L. Wu). 
In 1912, Hilbert proposed obtaining approximate solutions to the BE by a series expansion in Knudsen number ( $K n$, defined as the ratio of the molecular mean free path to the characteristic flow length). The Hilbert expansion inspired Sone to develop an asymptotic analysis of the BE at small $K n$, which helped reveal many interesting phenomena including the 'ghost effect' [1, 2]. In 1917, Chapman and Enskog (CE) obtained approximate solutions to the BE and calculated transport coefficients from first principles [3]. The first-order CE expansion recovers the Navier-Stokes equations, while the second- and third-order expansions lead to the Burnett and super-Burnett equations, respectively. Although the Burnett equations can be more accurate than the Navier-Stokes equations in some cases [4], they are rarely used nowadays due to their intrinsic instability to small-wavelength perturbations [5]. In 1949, by expanding the VDF into Gauss-Hermite polynomials, Grad proposed the moment method [6]. This has led to the development of regularized 13-moment [7] and 26-moment [8] equations, which have been successfully applied to rarefied gas flows up to $K n \sim 1$ in some circumstances. For large $K n$, a great number of moments are necessary but the convergence rate with increasing moment number is rather slow [9]. Moreover, multiple new boundary conditions are needed, so this approach it is not suitable to simulate highly rarefied gas flows.

For moderate and highly rarefied gas flows it is necessary to directly solve the BE. The direct simulation Monte Carlo (DSMC) method is the prevailing numerical technique for this [10]. In DSMC, the VDF is represented by a number of simulated particles that move in the computational domain and collide according to stochastic rules. The simulated particles automatically concentrate in regions where the VDF has large values, and discontinuities/fine structures in VDFs can be captured without difficulty. This method is efficient for hypersonic flows, however, its stochastic nature makes it not well suited for unsteady and low-speed flows. For instance, for a gas flow with a Mach number 0.001 (typically in micro-devices), about $10^{8}$ independent samples are needed to reduce the error to $1 \%$ when there are 100 simulated particles in a cell [11]. Also, DSMC is an inefficient method for near-continuum flows, since the spatial and temporal steps must be smaller than the molecular mean free path and collision time, respectively. Progress has been made to ease these difficulties: to resolve small macroscopic quantities, the information preserving [12] and low variance $[13,14]$ DSMC methods have been proposed; to simulate near-continuum flows, hybrid continuum-particle approaches $[15,16,17,18,19,20,21]$, time relaxed Monte Carlo method [22, 23], and particle-particle hybrid methods [24, 25] have been proposed. Recently, a new multiscale method has also been developed to simulate flows in geometries with high aspect ratio $[26,27,28]$.

Deterministic numerical methods $[29,30,31,32,33,34,35,36,37,38,39,40]$, based on the direct discretization of the $\mathrm{BE}$ in phase space, have distinct advantages in resolving small signals and low-speed flows as they are free of noise. Also, implicit time-marching can be employed to enlarge the time step [29, 30, 41, 42, 43]. Furthermore, compared to continuum/particle coupling, deterministic methods can be coupled more efficiently to macroscopic equations [44] such as the Navier-Stokes/moment equations and equations from the asymptotic kinetic analysis [2]. Among these deterministic solvers, however, only a few have been applied to spatially-inhomogeneous problems. The numerical kernel method [29, 30], in 
which the VDF is expanded into Laguerre polynomials for velocity components parallel to solid surfaces and quadratic finite-element functions for normal velocity component, provides accurate numerical results for many one-dimensional problems. As it allows non-uniform discretization of the normal velocity component, VDF discontinuities at large $K n$ are captured: for linearized Poiseuille and thermal transpiration flows, accurate results are produced up to $K n \sim 20$ for hard-sphere molecules [45]. The conservative projection-interpolation method, in which uniform Cartesian velocity discretization is usually employed, and post-collision velocities are interpolated to closest neighbors, has been proposed to solve the BE with realistic intermolecular potentials in three-dimensional space [32], with a numerical error of the order $\Delta v\left|f-f_{M}\right|$ ( $\Delta v$ is the velocity grid step, $f$ is the $\mathrm{VDF}$, and $f_{M}$ is the equilibrium VDF) [44]. Since VDFs at post-collision velocities are obtained by polynomial interpolation, this method is accurate at small $K n$ where the VDF is usually smooth (in a recent paper [46] an accuracy of $(\Delta v)^{2}$ has been demonstrated), but loses accuracy at large $K n$ where VDFs have discontinuities or steep variations. Discontinuous Galerkin methods, although able to capture VDF discontinuities, turn out to be feasible only for slightly rarefied subsonic gas flows [40] because of the high computational cost.

Recently, the fast spectral method (FSM) has been developed to numerically solve the BE for single-species monatomic gases [35, 47, 41, 42, 43]. It employs a Fourier-Galerkin discretization in velocity space, and handles binary collisions in the corresponding frequency space. The method is of spectral accuracy, and has a computational cost of $O\left(M^{2} N^{3} \log N\right)$, where $N$ is the number of frequency nodes in each frequency direction and $M^{2}$ is the number of discrete solid angles. Since it allows non-uniform velocity discretizations, and the number of frequency nodes can be far smaller than the number of velocity nodes [42, 43], the FSM is good at dealing with highly rarefied gas flows, where large numbers of velocity nodes are used to capture discontinuities/fine structures in the VDF: for linearized Poiseuille and thermal transpiration flows, accurate numerical results have been obtained even for $K n \sim 10^{6}$ (note that even at such high Knudsen numbers, collision cannot be ignored). The FSM can be 50 times faster than the low variance DSMC method [42], and it is very efficient in dealing with linearized oscillatory flows [43] and Rayleigh-Brillouin scattering of light by rarefied gases $[48,49]$.

Deterministic numerical methods for the BE for mixtures of monatomic gases are even more scarce. Compared to the single-species BE, the non-unitary mass ratio between different molecular species poses an additional difficulty. Accurate numerical results have previously only been reported for hard-sphere molecules in some simple spatial configurations $[50,51,52,53,54,55]$. For general cases, a multipoint conservative projectioninterpolation method has been developed for an arbitrary ratio of molecular masses [56], but its accuracy is not known, especially at large Knudsen numbers. Recently, a spectralLagrangian method with a computational cost of $O\left(m_{r}^{3} N^{6}\right)$ has been proposed (where $m_{r}$ is the mass ratio of the heavier to the lighter species), and a normal shock wave in a binary mixture with a mass ratio of about 2 has been simulated [57]. As this method uses the same velocity discretization for each component, it cannot be applied to mixtures with large mass ratios, since the computational cost and storage are $m_{r}^{3}$ and $m_{r}^{3 / 2}$ times larger than that for the single-species BE, respectively. As an example, calculation of an argon-helium 
mixture with the mass ratio $m_{r} \approx 10$ requires a 30 -times increase in velocity grid points, and a 1000-times increase in computational time.

Because of the excellent performance of the FSM, in this paper we develop an efficient and accurate FSM for gas mixtures. The rest of this paper is organized as follows. In Section 2, the BE is introduced. The fast spectral approximation to the Boltzmann collision operator with general forms of the collision kernel is presented in Section 3, and its accuracy is assessed in Section 4 by comparing numerical results with analytical solutions for the spatiallyhomogeneous relaxation of pseudo-Maxwell molecules. In Section 5, an efficient algorithm is proposed to deal with gas mixtures of large molecular mass ratio. In Section 6, an iteration scheme is used to find the steady-state solutions of spatially-inhomogeneous problems, and various comparisons with results from the numerical kernel and DSMC methods are made for normal shocks and planar Fourier/Couette flows. A two-dimensional temperature-driven flow is also investigated. In Section 7 , we conclude with a summary of our proposed numerical method, and outline future perspectives.

\section{Boltzmann equation for a binary gas mixture}

Consider a binary gas mixture of components $\mathrm{A}$ and $\mathrm{B}$, where the mass of a molecule of component $\mathrm{A}$ is $m^{A}$, and that of component $\mathrm{B}$ is $m^{B}$. Without loss of generality, we assume $m^{A} \geq m^{B}$. Let $f^{A}(t, x, v)$ and $f^{B}(t, x, v)$ be respectively the VDFs of the components A and $\mathrm{B}$ of the molecular velocity $v=\left(v_{1}, v_{2}, v_{3}\right)$ at spatial location $x=\left(x_{1}, x_{2}, x_{3}\right)$ and time $t$. In the absence of external forces, the BEs for a binary gas mixture of monatomic molecules take the following forms:

$$
\frac{\partial f^{\imath}}{\partial t}+v \cdot \frac{\partial f^{\imath}}{\partial x}=\sum_{\jmath=A, B} Q^{\imath \jmath}\left(f^{\imath}, f^{\jmath}\right) \quad(\imath=A, B) .
$$

Here $Q^{\imath \jmath}\left(f^{\imath}, f^{\jmath}\right)$ are the self-collision operators when $\imath=\jmath$, and cross-collision operators when $\imath \neq \jmath$. The collision operators are local in time and space and consist of the gain part $Q^{\imath \jmath+}$ and the loss part $Q^{\imath \jmath-}$. For simplicity, $t$ and $x$ are omitted in writing the collision operators

$$
Q^{\imath \jmath}\left(f^{\imath}, f^{\jmath}\right)=\underbrace{\int_{\mathbb{R}^{3}} \int_{\mathbb{S}^{2}} C^{\imath \jmath}(\theta,|u|) f^{\jmath}\left({ }^{\prime} v_{*}^{\imath \jmath}\right) f^{\imath}\left({ }^{\prime} v^{\imath \jmath}\right) d \Omega d v_{*}}_{Q^{\imath \jmath}+}-\underbrace{\nu^{\imath \jmath}\left(f^{\jmath}\right) f^{\imath}(v)}_{Q^{\imath \jmath-}},
$$

where

$$
\nu^{\imath \jmath}\left(f^{\jmath}\right)=\int_{\mathbb{R}^{3}} \int_{\mathbb{S}^{2}} C^{\imath \jmath}(\theta,|u|) f^{\jmath}\left(v_{*}\right) d \Omega d v_{*}
$$

are the collision frequencies, $v$ and $v_{*}$ are the pre-collision velocities of molecules of sorts $\imath$ and $\jmath$, respectively, while ${ }^{\prime} v^{\imath \jmath},{ }^{\prime} v_{*}^{\imath \jmath}$ are the corresponding post-collision velocities. Conservation of momentum and energy yield the following relations

$$
{ }^{\prime} v^{\imath \jmath}=v+\frac{m^{\jmath}}{m^{\imath}+m^{\jmath}}(|u| \Omega-u), \quad{ }^{\prime} v_{*}^{\imath \jmath}=v_{*}-\frac{m^{\imath}}{m^{\imath}+m^{\jmath}}(|u| \Omega-u),
$$


with $u=v-v_{*}$ being the relative pre-collision velocity, $\Omega$ the unit vector in the sphere $\mathbb{S}^{2}$ having the same direction as the relative post-collision velocity, and $\theta$ is the deflection angle between the two relative velocities, i.e. $\cos \theta=\Omega \cdot u /|u|, 0 \leq \theta \leq \pi$.

The collision kernels $C^{\imath \jmath}$ are always non-negative. In general, they depend on the modulus of the relative pre-collision velocities and the deflection angle. When hard-sphere molecules are considered, we have

$$
C^{A A}=\frac{\left(d_{m}^{A}\right)^{2}}{4}|u|, \quad C^{B B}=\frac{\left(d_{m}^{B}\right)^{2}}{4}|u|, \quad C^{A B}=C^{B A}=\frac{\left(d_{m}^{A}+d_{m}^{B}\right)^{2}}{16}|u|,
$$

where $d_{m}^{A}$ and $d_{m}^{B}$ are the molecular diameters of components $\mathrm{A}$ and $\mathrm{B}$, respectively. For general intermolecular potentials, the calculation of the collision kernel is complicated. In the following sections, we present the FSM for the Boltzmann collision operator with general forms of collision kernels, but in detailed calculations we only consider the following special collision kernels:

$$
C^{\imath \jmath}=C_{0}^{\imath \jmath} \sin ^{\alpha^{\imath \jmath}+\gamma^{\imath \jmath}-1}\left(\frac{\theta}{2}\right) \cos ^{-\gamma^{\imath \jmath}}\left(\frac{\theta}{2}\right)|u|^{\alpha^{\imath \jmath}},
$$

where symmetry requires $C_{0}^{A B}=C_{0}^{B A}, \alpha^{A B}=\alpha^{B A}$, and $\gamma^{A B}=\gamma^{B A}$. These collision kernels can be used to recover the coefficients of viscosity, diffusion, and thermal conductivity for inverse power-law potentials. For more information, see Appendix A.

\section{Fast spectral method for the cross-collision operators}

The approximation of the self-collision operator (with special forms of the collision kernel) by using FSM has been studied extensively [34, 36, 41, 42]. Here we consider the numerical approximation of cross-collision operators with general forms of the collision kernel.

In FSM, VDFs are periodized on the velocity domain. For simplicity, we assume the velocity domains of $f^{A}$ and $f^{B}$ are the same, say, $\mathcal{D}_{L}=[-L, L]^{3}$. The velocity bound $L$ should be chosen as a compromise between the resolution and the aliasing error. Suppose $\mathcal{B}_{S}$, a sphere of radius $S$ centered on the origin, is the support of the VDFs. According to Ref. [34], $L=(3+\sqrt{2}) S / 2$ is chosen to avoid the aliasing error caused in the periodization of VDFs. The truncated velocity space can be discretized either uniformly or non-uniformly, but frequency space must be discretized uniformly. Suppose the frequency components are denoted by

$$
\xi=\left(\xi_{1}, \xi_{2}, \xi_{3}\right)=\left(j_{1}, j_{2}, j_{3}\right) \frac{\pi}{L}=j \frac{\pi}{L},
$$

where $j_{k} \in\left[-N_{k} / 2,-N_{k} / 2+1, \cdots, N_{k} / 2-1\right]$ and $N_{k}$ is the number of frequency components in the $k$-th frequency direction. The VDFs are then approximated by the truncated Fourier series,

$$
\begin{aligned}
f^{\imath}(v) & =\sum_{j=-\left(N_{1}, N_{2}, N_{3}\right) / 2}^{\left(N_{1}, N_{2}, N_{3}\right) / 2-1} \hat{f}_{j}^{\imath} \exp \left(i \xi_{j} \cdot v\right), \\
\hat{f}_{j}^{\imath} & =\frac{1}{(2 L)^{3}} \int_{\mathcal{D}_{L}} f^{\imath}(v) \exp \left(-i \xi_{j} \cdot v\right) d v
\end{aligned}
$$


where $i$ is the imaginary unit.

With the basic identity $\int_{\mathbb{S}^{2}}|u| F\left(\frac{|u| \Omega-u}{2}\right) d \Omega=4 \int_{\mathbb{R}^{3}} \delta\left(y \cdot u+|y|^{2}\right) F(y) d y$, where $\delta$ is the Dirac delta function, the cross-collision operator (2) can be rewritten in the Carleman representation as

$$
\begin{aligned}
Q^{\imath \jmath} & =\int_{\mathbb{R}^{3}} \int_{\mathbb{S}^{2}} C^{\imath \jmath}\left[f^{\jmath}\left(v_{*}-\left(1-b^{\imath \jmath}\right) \frac{|u| \Omega-u}{2}\right) f^{\imath}\left(v+a^{\imath \jmath} \frac{|u| \Omega-u}{2}\right)-f^{\jmath}\left(v_{*}\right) f^{\imath}(v)\right] d \Omega d v_{*} \\
& =4 \int_{\mathbb{R}^{3}} \int_{\mathbb{R}^{3}} \Theta(\theta,|u|) \delta\left(y \cdot u+|y|^{2}\right)\left[f^{\jmath}\left(v_{*}-\left(1-b^{\imath \jmath}\right) y\right) f^{\imath}\left(v+a^{\imath \jmath} y\right)-f^{\jmath}\left(v_{*}\right) f^{\imath}(v)\right] d y d v_{*} \\
& =4 \int_{\mathbb{R}^{3}} \int_{\mathbb{R}^{3}} \Theta(\theta,|u|) \delta(y \cdot z)\left[f^{\jmath}\left(v+z+b^{\imath \jmath} y\right) f^{\imath}\left(v+a^{\imath \jmath} y\right)-f^{\jmath}(v+y+z) f^{\imath}(v)\right] d y d z,
\end{aligned}
$$

where

$$
a^{\imath \jmath}=\frac{2 m_{\jmath}}{m_{\imath}+m_{\jmath}}, \quad b^{\imath \jmath}=\frac{m_{\jmath}-m_{\imath}}{m_{\imath}+m_{\jmath}},
$$

and $\Theta(\theta,|u|)=C^{\imath \jmath} /|u|$ is the differential cross-section, which is solely determined by the intermolecular potential.

Note that in the above manipulations we have used the transformations $y=(|u| \Omega-u) / 2$ and $z=v_{*}-v-y=-u-y$. Therefore, $u=-y-z$ and the deflection angle $\theta$ satisfies $\cos \theta=$ $\Omega \cdot u /|u|=-(y-z) \cdot(y+z) /|y+z|^{2}$. Since $y \perp z$, we have $\cos \theta=\left(|z|^{2}-|y|^{2}\right) /\left(|y|^{2}+|z|^{2}\right)$, which leads to $\theta=2 \arctan (|y| /|z|)$, and the differential cross-section becomes a function of $|y|$ and $|z|$ only:

$$
\Theta(\theta,|u|) \equiv \Theta^{\prime}(|y|,|z|)
$$

The collision operator (10) is simplified to $4 \int_{\mathbb{R}^{3}} \int_{\mathbb{R}^{3}} \delta(y \cdot z) \Theta^{\prime}(|y|,|z|)\left[f^{\jmath}\left(v+z+b^{\imath \jmath} y\right) f^{\imath}(v+\right.$ $\left.\left.a^{\imath \jmath} y\right)-f^{\jmath}(v+y+z) f^{\imath}(v)\right] d y d z$. Since the VDFs have the support $S$, the relative velocity $|u|=|y+z| \leq 2 S$. Therefore, the infinite integral region for $y$ and $z$ can be reduced to $\mathcal{B}_{R}$, with

$$
R=\sqrt{2} S=\frac{2 \sqrt{2}}{3+\sqrt{2}} L
$$

which results in the truncated collision operator

$$
Q^{\imath \jmath}=4 \int_{\mathcal{B}_{R}} \int_{\mathcal{B}_{R}} \delta(y \cdot z) \Theta^{\prime}(|y|,|z|)\left[f^{\jmath}\left(v+z+b^{\imath \jmath} y\right) f^{\imath}\left(v+a^{\imath \jmath} y\right)-f^{\jmath}(v+y+z) f^{\imath}(v)\right] d y d z
$$

Expanding the truncated collision operator as a Fourier series, we find that the $j$-th mode of the truncated collision operator is related to the Fourier coefficients $\hat{f}^{\imath}$ and $\hat{f} \jmath$ as follows:

$$
\widehat{Q}_{j}^{\imath \jmath}=\sum_{\substack{l+m=j \\ l, m=-\left(N_{1}, N_{2}, N_{3}\right) / 2}}^{\left.N_{1}, N_{2}, N_{3}\right) / 2-1} \hat{f}_{l}^{\imath} \hat{f}_{m}^{\jmath} \beta_{1}^{\imath \jmath}(l, m)-\hat{f}_{l}^{\imath} \hat{f}_{m}^{\jmath} \beta_{2}(m),
$$

where $l=\left(l_{1}, l_{2}, l_{3}\right), m=\left(m_{1}, m_{2}, m_{3}\right)$, the kernel mode $\beta_{1}^{\imath \jmath}(l, m)$ is

$$
\beta_{1}^{\imath \jmath}(l, m)=4 \int_{\mathcal{B}_{R}} \int_{\mathcal{B}_{R}} \delta(y \cdot z) \Theta^{\prime}(|y|,|z|) \exp \left[i a^{\imath \jmath} \xi_{l} \cdot y+i \xi_{m} \cdot\left(z+b^{\imath \jmath} y\right)\right] d y d z
$$


and $\beta_{2}(m)$ is equal to $\beta_{1}(m, m)$ when $a^{\imath \jmath}=1$ and $b^{\imath \jmath}=0$.

Note that the second term on the right hand side of Eq. (15) can be calculated by FFTbased convolution with a computational cost $O\left(N^{3} \log N\right)$, where $N$ is the same order as $N_{1}, N_{2}$ and $N_{3}$. For the first term, however, direct calculation has a cost $O\left(N^{6}\right)$. The main achievement of the original FSM [35] is the decomposition of $\Theta^{\prime}(|y|,|z|)$ into $\Theta_{1}^{\prime}(|y|) \Theta_{2}^{\prime}(|z|)$, so that $\xi_{l}$ and $\xi_{m}$ are separated from $\beta_{1}(l, m)$ and Eq. (15) can also be calculated effectively by FFT-based convolution. However, for general intermolecular potentials, this decomposition is not possible. Here we propose a new way to separate $\xi_{l}$ and $\xi_{m}$ in $\beta_{1}(l, m)$ for general forms of $\Theta^{\prime}(|y|,|z|)$.

The right-hand side of Eq. (16) can be expressed in spherical coordinates as $4 \iint \delta(e$. $\left.e^{\prime}\right) \int_{0}^{R} \int_{0}^{R} \rho \rho^{\prime} \Theta^{\prime}\left(\rho, \rho^{\prime}\right) \cos \left[\rho\left(a^{\imath \jmath} \xi_{l}+b^{\imath \jmath} \xi_{m}\right) \cdot e\right] \cos \left(\rho^{\prime} \xi_{m} \cdot e^{\prime}\right) d \rho^{\prime} d \rho d e^{\prime} d e$, where $e, e^{\prime}$ are vectors in the unit sphere $\mathbb{S}^{2}$. Inspired by work on the FSM for quantum Boltzmann equations [58], the integral with respect to $\rho$ is approximated by Gauss-Legendre quadrature. Suppose $\rho_{r}$ and $\omega_{r}\left(r=1,2, \cdots, M_{2}\right)$ are the abscissas and weights of the Gauss-Legendre quadrature for $\rho$ in the region $[0, R]$, we have

$$
\beta_{1}^{\imath \jmath}(l, m)=\int I^{\imath \jmath}\left(\xi_{l}, \xi_{m}, e\right)\left[\int \delta\left(e \cdot e^{\prime}\right) \phi\left(\xi_{m}, e^{\prime}\right) d e^{\prime}\right] d e,
$$

where $\phi\left(\xi_{m}, e^{\prime}\right)=2 \int_{0}^{R} \rho^{\prime} \Theta^{\prime}\left(\rho_{r}, \rho^{\prime}\right) \cos \left(\rho^{\prime} \xi_{m} \cdot e^{\prime}\right) d \rho^{\prime}$ and

$$
I^{\imath \jmath}\left(\xi_{l}, \xi_{m}, e\right)=2 \sum_{r=1}^{M_{2}} \omega_{r} \rho_{r} \cos \left[\rho_{r}\left(a^{\imath \jmath} \xi_{l}+b^{\imath \jmath} \xi_{m}\right) \cdot e\right] .
$$

After some algebraic manipulation (see descriptions from Eq. (34) to Eq. (38) in Ref. [41]), we have

$$
\beta_{1}^{\imath \jmath}(l, m)=\frac{4 \pi^{2}}{M^{2}} \sum_{p, q=1}^{M-1, M} I^{\imath \jmath}\left(\xi_{l}, \xi_{m}, e_{\theta_{p}, \varphi_{q}}\right) \psi\left(\sqrt{\left|\xi_{m}\right|^{2}-\left(\xi_{m} \cdot e_{\theta_{p}, \varphi_{q}}\right)^{2}}\right) \sin \theta_{p}
$$

where $e_{\theta_{p}, \varphi_{q}}=\left(\sin \theta_{p} \cos \varphi_{q}, \sin \theta_{p} \sin \varphi_{q}, \cos \theta_{p}\right), \theta_{p}=p \pi / M$, and $\varphi_{q}=q \pi / M$, while

$$
\psi(s)=2 \pi \int_{0}^{R} \rho^{\prime} \Theta^{\prime}\left(\rho_{r}, \rho^{\prime}\right) J_{0}\left(\rho^{\prime} s\right) d \rho^{\prime},
$$

with $J_{0}$ being the zeroth-order Bessel function of first kind.

To separate $\xi_{l}$ and $\xi_{m}, I^{\imath \jmath}\left(\xi_{l}, \xi_{m}, e_{\theta_{p}, \varphi_{q}}\right)$ is expanded as follows:

$$
\begin{aligned}
I^{\imath \jmath}\left(\xi_{l}, \xi_{m}, e_{\theta_{p}, \varphi_{q}}\right)=2 \sum_{r=1}^{M_{2}} \omega_{r} \rho_{r}[ & \cos \left(\rho_{r} a^{\imath \jmath} \xi_{l} \cdot e_{\theta_{p}, \varphi_{q}}\right) \cos \left(\rho_{r} b^{\imath \jmath} \xi_{m} \cdot e_{\theta_{p}, \varphi_{q}}\right) \\
& \left.-\sin \left(\rho_{r} a^{\imath \jmath} \xi_{l} \cdot e_{\theta_{p}, \varphi_{q}}\right) \sin \left(\rho_{r} b^{\imath \jmath} \xi_{m} \cdot e_{\theta_{p}, \varphi_{q}}\right)\right] .
\end{aligned}
$$

Since $\xi_{l}$ and $\xi_{m}$ are now separated, Eq. (15) can be calculated with a computational cost of $O\left(M^{2} M_{2} N^{3} \log N\right)$ through FFT-based convolution. From Eq. (18) we see that the cosine 
function oscillates $O(N)$ times, so $M_{2}$ should be roughly of the order of $N$. Therefore, the overall computational cost is $O\left(M^{2} N^{4} \log N\right)$. Although slower than the original FSM [35], the present FSM is applicable for general intermolecular potentials, and its efficiency is still better than conventional spectral methods with the computational cost $O\left(N^{6}\right)$.

When $\widehat{\mathcal{Q}}^{\imath \jmath}$ is obtained, the collision operator $\mathcal{Q}^{\imath \jmath}$ can be obtained through FFT:

$$
\mathcal{Q}^{\imath \jmath}(v)=\sum_{j} \widehat{\mathcal{Q}}_{j}^{\imath \jmath} \exp \left(i \xi_{j} \cdot v\right) .
$$

Note that the procedure in deriving this FSM for monatomic gas mixtures is similar to that for a single-species Boltzmann collision operator [35], so it can be proved that the FSM presented here conserves mass, while the errors in achieving momentum and energy conservation are spectrally small. These errors, however, can be removed using the method of Lagrangian multipliers [37, 57].

\section{Accuracy analysis of the fast spectral method}

We assess the accuracy of our newly-developed FSM by comparing its numerical results with the analytical Bobylev-Krook-Wu (BKW) solutions for the space-homogeneous relaxation of pseudo-Maxwell molecules when the collision kernel is $C^{\imath \jmath}=C_{0}^{\imath \jmath} \sin ^{-1}(\theta / 2)$. For simplicity, we take $k_{B}=1$, and set the equilibrium temperature to be 2 . The exact BKW solutions can be written as follows [59, 60]:

$$
f_{B K W}^{\imath}(v, t)=n^{\imath}\left(\frac{m^{\imath}}{2 \pi K}\right)^{3 / 2} \exp \left(-\frac{m^{\imath}|v|^{2}}{2 K}\right)\left(1-3 r p^{\imath}+\frac{r p^{\imath}}{K} m^{\imath}|v|^{2}\right) \quad(\imath=A, B),
$$

where

$$
\begin{aligned}
p^{A} & =\frac{4 n^{B}}{5}\left[2 C_{0}^{B B}-m_{0} C_{0}^{A B}\left(5-3 m_{0}\right)\right], \quad p^{B}=\frac{4 n^{A}}{5}\left[2 C_{0}^{A A}-m_{0} C_{0}^{A B}\left(5-3 m_{0}\right)\right], \\
m_{0} & =\frac{4 m^{A} m^{B}}{\left(m^{A}+m^{B}\right)^{2}}, \quad r=\frac{\widetilde{A}}{\widetilde{A} \exp \left[4 \pi \widetilde{A}\left(t+t_{0}\right)\right]-\widetilde{B}}, \quad K=\frac{n^{A}+n^{B}}{n^{A}+n^{B}+2\left(n^{A} p^{A}+n^{B} p^{B}\right) r}, \\
\widetilde{A} & =\frac{4 n^{A} C_{0}^{A A}+2 n^{B} m_{0} C_{0}^{A B}\left(5-3 m_{0} p^{B} / p^{A}\right)}{15}, \quad \widetilde{B}=\frac{8 n^{A} C_{0}^{A A} p_{1}+4 n^{B} m_{0} C_{0}^{A B}\left(5-3 m_{0}\right) p^{B}}{15} .
\end{aligned}
$$

Additional conditions should be satisfied for the existence of exact BKW solutions:

- exact solution of type I exists when $p^{A}=p^{B}$, i.e. there is no energy exchange between the two components. This solution exists only for special values of the relative density:

$$
\frac{n^{B}}{n^{A}}=\frac{2 C_{0}^{A A}-m_{0}\left(5-3 m_{0}\right) C_{0}^{A B}}{2 C_{0}^{B B}-m_{0}\left(5-3 m_{0}\right) C_{0}^{A B}}
$$

- exact solution of type II exists for arbitrary values of $n^{A}$ and $n^{B}$ when the following relation is satisfied:

$$
\frac{1}{2 C_{0}^{A A}-m_{0} C_{0}^{A B}\left(5-3 m_{0}\right)}+\frac{1}{2 C_{0}^{B B}-m_{0} C_{0}^{A B}\left(5-3 m_{0}\right)}=\frac{1}{3 m_{0}^{2} C_{0}^{A B}} .
$$



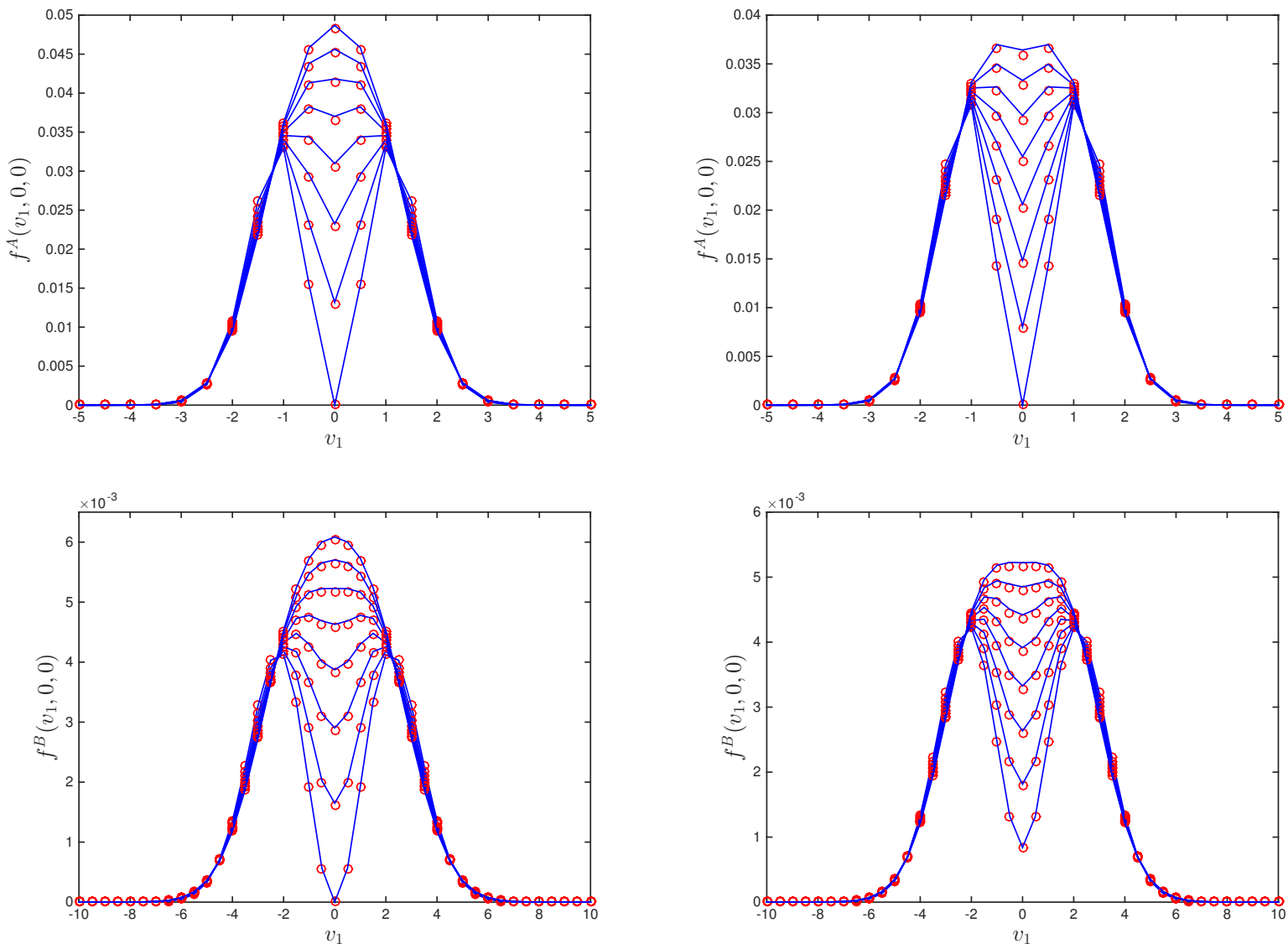

Figure 1: The time evolution of VDFs in the spatially-homogeneous relaxation problem for pseudo-Maxwell molecules. Left (right) column: type I (II) solutions. Solid lines represent exact BKW solutions, while circles are the numerical solutions from our FSM. In each figure, from bottom to top (near $v_{1}=0$ ), the time for each line is $(0,1,2, \cdots, 7) \times 0.2$. In the left figures, parameters are $C_{0}^{B B}=C_{0}^{A A}=4 C_{0}^{A B}=1 / 4 \pi$, and $n^{A}=n^{B}=1$. In the right figures, parameters are $C_{0}^{A B}=1 / 16 \pi, C_{0}^{B B}=C_{0}^{A A}=C_{0}^{A B}\left(3 m_{0}^{2}+5 m_{0}\right) / 2$, $n^{A}=0.95$, and $n^{B}=1$. The initial time is $t_{0}=\max \left\{\log \left[\left(B+3 A p_{1}\right) / A\right], \log \left[\left(B+3 A p_{2}\right) / A\right]\right\} / 4 A \pi$.

Figure 1 depicts the relaxation-to-equilibrium process of the two VDFs for pseudoMaxwell molecules with a mass ratio $m^{A} / m^{B}=4$. In numerical simulations, the number of solid angle discretizations is $M(M-1)=20$ and the velocity domain $[-L, L)^{3}$ with $L=16$ is discretized by $64 \times 64 \times 64$ uniform grid points. For $M_{2}=7$, the obtained VDFs from the FSM almost coincide with the analytical BKW solutions.

Relative errors of the zeroth-, second-, fourth-, and sixth-order moments of VDFs as functions of the time are shown in Fig. 2. Odd-order moments are not included because they are zero due to symmetry. From the figure we can see that, at $N=64$, mass is conserved, as relative errors in the density are negligible. Energy (temperature) is not conserved, but the maximum deviation from the equilibrium value is about $10^{-5}$. Although the relative error increases with the order of moment, deviations of the sixth-order moment $\int f^{\imath} v_{1}^{6} d v$ from analytical solutions are still small. Relative errors for the type II solution exhibit similar

9 

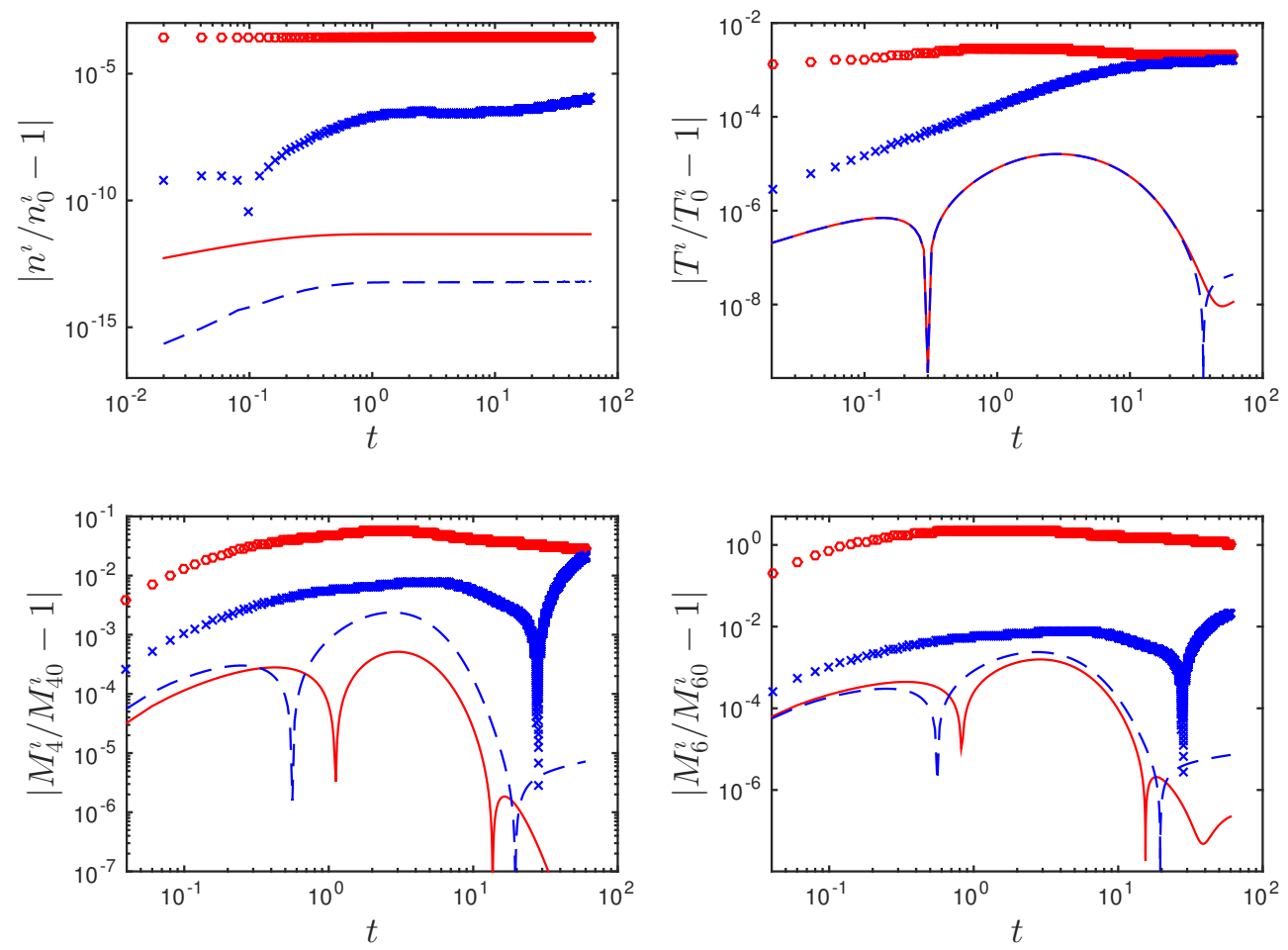

Figure 2: The time evolution of relative errors in even-order moments of the VDFs. Parameters are the same as for the type I solution in Fig. 1. The moments with subscript 0 are calculated from the analytical BKW solution. Solid and dashed lines: components $\mathrm{A}$ and $\mathrm{B}$ at $N=64$, respectively. Circles and crosses: components $\mathrm{A}$ and $\mathrm{B}$ at $N=32$, respectively.

behaviors.

To demonstrate the grid dependence of the FSM, we run another simulation on a coarser grid with $N=32$. We see from Fig. 2 that the accuracy is greatly reduced, especially for the component A. This is because, for low $N$, some high frequency components of $\hat{f}^{A}$ are not included in the calcualtion, so even the mass of the component $\mathrm{A}$ is not conserved. The spectral accuracy of the present FSM is observed as relative errors are reduced by several orders of magnitude when $N$ is doubled.

\section{An efficient algorithm for large mass ratios}

In Section 4 we used the same velocity discretization for both VDFs. This is acceptable for mass ratios less than 2 , but it becomes computationally inefficient for large mass ratios, as the computer memory usage of FSM is proportional to $m_{r}^{3 / 2}$, while the computational cost is proportional to $m_{r}^{2}$. Here we propose a more efficient algorithm to deal with large-mass-ratio problems.

Before starting, we demonstrate how the cross-collision operators are calculated in Section 4 when using the same velocity discretization for both VDFs. Without loss of generality, the VDFs are chosen to be those used in the left column of Fig. 1 at $t=0$. The first step 

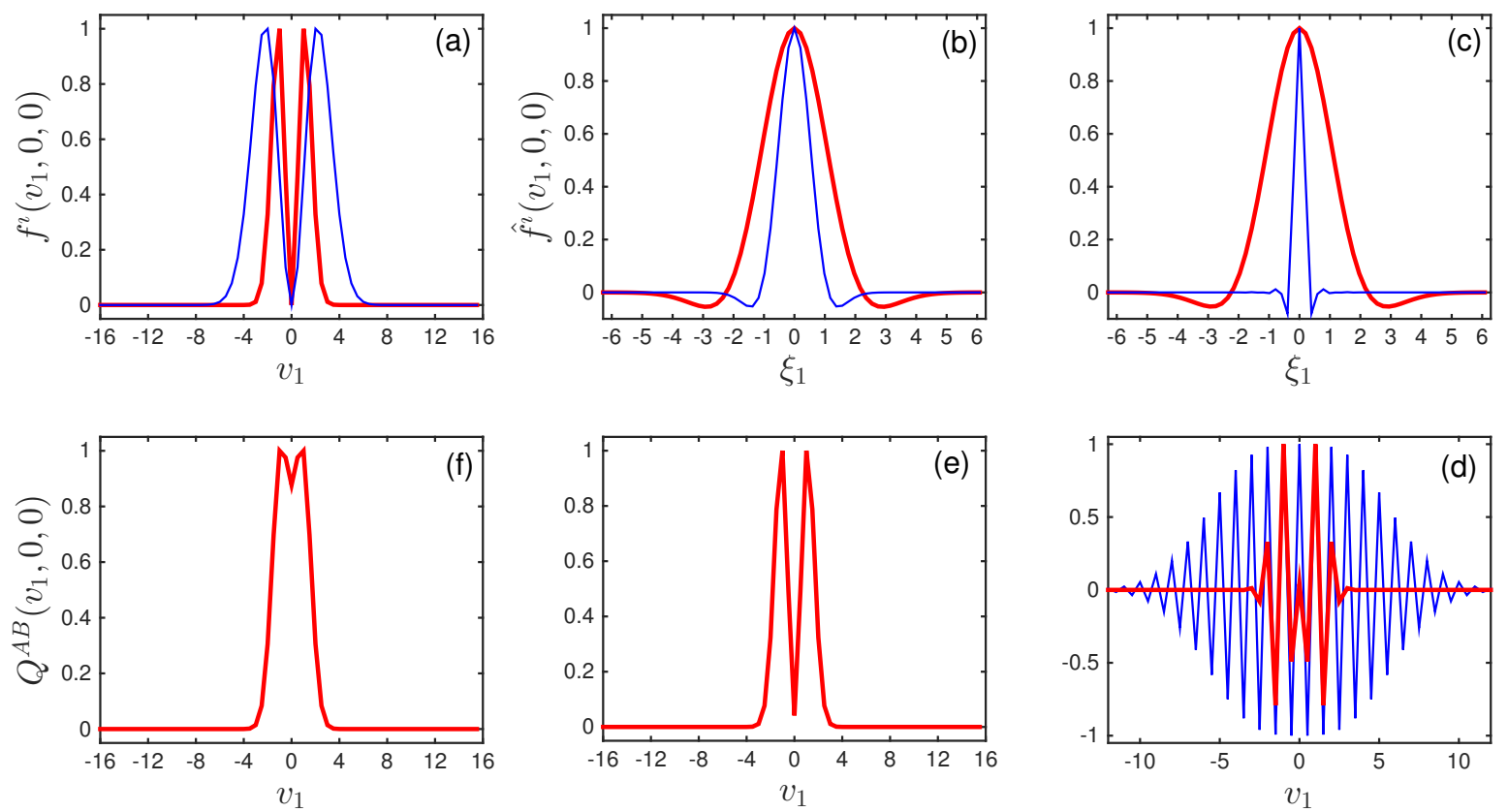

Figure 3: The process of obtaining the Boltzmann cross-collision operator $Q^{A B}$. (a) the VDFs; (b) the spectra of the VDFs; (c) weighted spectra; (d) spectra of the weighted spectra; (e) the product of the two lines in (d); (f) $Q^{A B}$. Thick red lines are for component $\mathrm{A}$, while thin blue lines are for B. Each line is normalized by its maximum value.

is to generate the spectra of VDFs; in Matlab, this is achieved by $\hat{f}^{\imath}=\mathcal{S F}^{-1} \mathcal{S} f^{\imath}$, where $\mathcal{S}$ is the fftshift function and $\mathcal{F}^{-1}$ is the inverse FFT function. The obtained spectra are shown in Fig. 3(b). Then each spectrum is multiplied by the corresponding weight function in the kernel mode $\beta_{1}^{A B}\left(\xi_{l}, \xi_{m}\right)$, see Eqs. (15), (19), and (21). The weighted spectra are shown in Fig. 3(c) when $\theta_{p}, \varphi_{q}=\pi / 5$ and $\rho_{r}=0.8582$. Figure 3(d) shows the spectra of the weighted spectra, which are obtained by applying the FFT and fftshift functions to the weighted spectra. The product of the two spectra (now in velocity space) from Fig. 3(d) is shown in Fig. 3(e), which is one of the components of the cross-collision operator $Q^{A B}$. Summing up all components, we obtain $Q^{A B}$, as shown in Fig. 3(f). A similar process to calculate the cross-collision operator $Q^{B A}$ is shown in Fig. 4.

The most time-consuming part of FSM is the calculation of the spectrum of the weighted spectra, as in Fig. 3(c,d) and Fig. 4(c,d), because this has to be repeated $M^{2} M_{2}$ times, each time with a computational cost of $O\left(N^{3} \log N\right)$, where $N=64$ in this specific case. From Figs. 3(c) and 4(c) we see that the weighted spectra for the B-component is restricted to the central frequency region $-3<\xi_{1}<3$ because of its small mass, which means that it is sufficient to use the central 32 frequency components to obtain its spectrum. Also, the weighted spectrum of the A-component in Fig. 3(c) is quite smooth, so fewer frequency components with a larger frequency step can be used. Further, for the VDF of the Bcomponent in Fig. 3(d), we see that only the central velocity region $-8<v_{1}<8$ contributes to $Q^{A B}$. These findings enable us to reduce the computational cost.

The first goal of an algorithm to improve the efficiency of the FSM is to remove the mass 

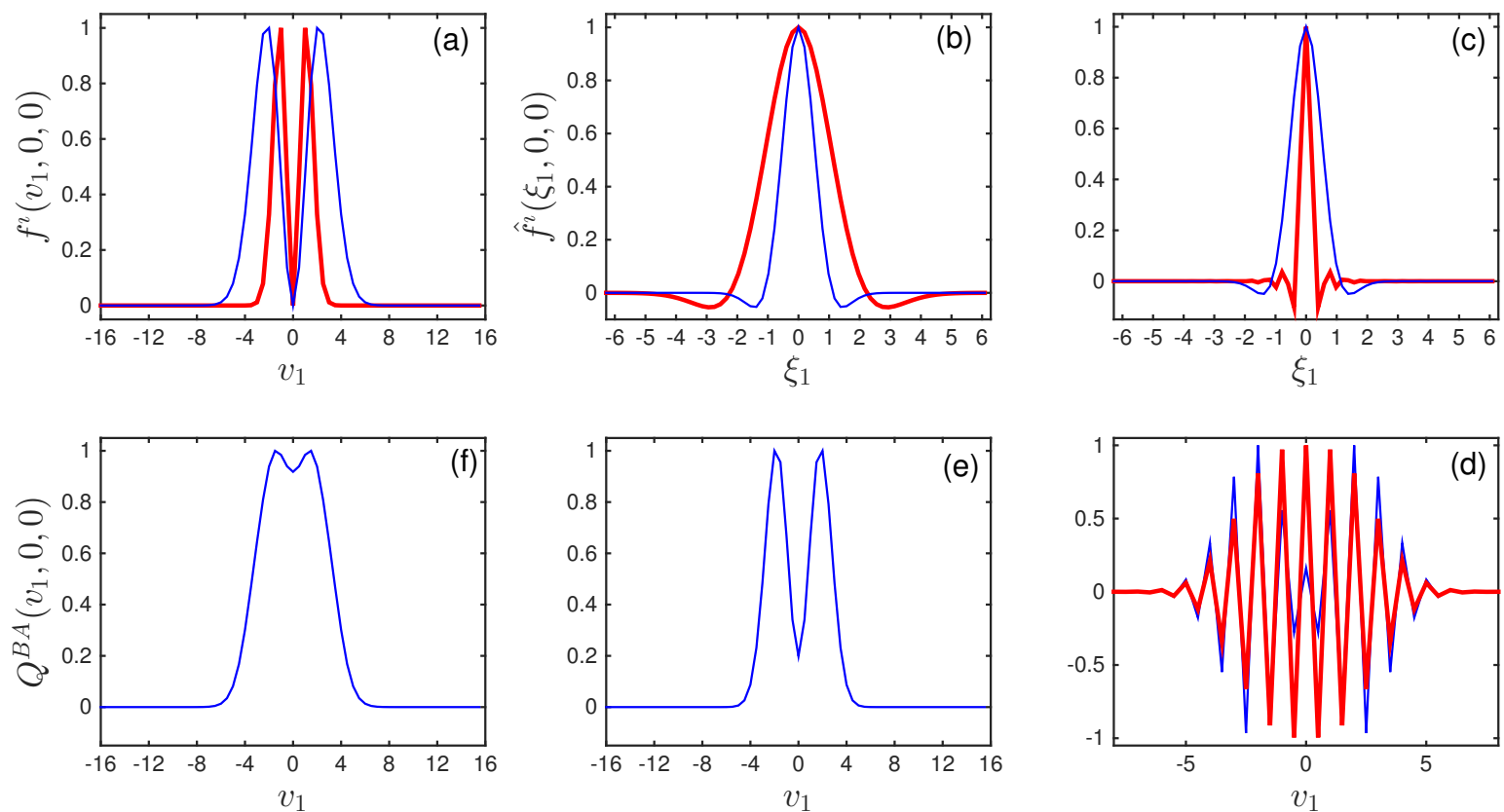

Figure 4: As Fig. 3, except here for the Boltzmann cross-collision operator $Q^{B A}$.

ratio dependence of the memory usage. Suppose the velocity region of the A-component $\left[-L_{A}, L_{A}\right]^{3}$ is uniformly discretized by $N_{1} \times N_{2} \times N_{3}$ points and the corresponding frequency space is uniformly discretized by the same number of points:

$$
v^{A}=\frac{2 L_{A}}{N_{v}} j, \quad \xi^{A}=\frac{\pi}{L_{A}} j .
$$

We then choose $M_{A B}$ to be the closest integer to $\sqrt{m_{r}}$. The velocity and frequency spaces of the B-component are discretized as:

$$
v^{B}=M_{A B} v^{A}, \quad \xi^{B}=\frac{\xi^{A}}{M_{A B}} .
$$

The second goal is to improve the efficiency of the calculation of cross-collision operators. Taking into account that the spectrum of component $\mathrm{B}$ is confined to the central frequency region, and the kernel mode for component $\mathrm{A}$ is smooth (described by the sine and cosine functions), an efficient algorithm for calculating the cross-collision operator $Q^{A B}$ is:

- calculate the Fourier spectra of VDFs $f^{A}$ and $f^{B}$ in the discretized frequency spaces $\xi^{A}$ and $\xi^{B}$, respectively.

- given values of $\theta_{p}, \varphi_{q}$, and $r$ :

1. calculate the spectrum of the weighted spectrum: $t_{1}=\mathcal{S F}\left[\hat{f}_{l}^{A} \beta_{1}^{A B}(l)\right]$, at a computational cost of $O\left(N^{3} \log N\right)$. Note that $\beta_{1}^{A B}(l)$ is the kernel mode $\beta_{1}^{A B}(l, m)$, which involves only $l$. 
2. let $t_{2}=\hat{f}_{l}^{B} \beta_{1}^{A B}(m)$.

3. zero-pad $t_{2}$ to the size of $M_{A B} N_{1} \times N_{2} \times N_{3}$ in the first frequency $\left(\xi_{1}\right)$ direction, calculate its frequency spectrum in $\xi_{1}$ direction through FFT (by applying $\mathcal{F}$ and $\mathcal{S}$ to $t_{2}$ in $\xi_{1}$ direction), and assign the central $N_{1} \times N_{2} \times N_{3}$ frequency components to $t_{2}$. The computational cost is $O\left(M_{A B} N^{3} \log N\right)$.

4. repeat step 3 in the $\xi_{2}$ and $\xi_{3}$ directions.

5. let $t_{3}=t_{1} t_{2}$.

- sum up all $t_{3}$ for each set of $\theta_{p}, \varphi_{q}, r$, and the final result is the gain part of the collision operator $Q^{A B}$, that is, $Q^{A B+}$.

- the loss part of the collision operator $Q^{A B}$ is calculated in exactly the same way as that for the loss part of $Q^{B B}$, see Appendix B in Ref. [41].

Considering that the spectrum of component $\mathrm{B}$ is confined to the central frequency region, and the kernel mode for component A decays quickly, the calculation of the cross-collision operator $Q^{B A}$ is:

- refine $\hat{f}^{A}$ by FFT interpolation, such that its frequency step is the same as that of component B. The computational cost is $O\left(M_{A B} N^{3} \log N\right)$.

- select central frequency components of $\hat{f}_{m}^{A}$ : the total number of chosen frequency components is $\left(M_{e x, 1} N_{1}\right)\left(M_{e x, 2} N_{2}\right)\left(M_{e x, 3} N_{3}\right)$, where the integer $M_{e x, k}$ satisfies $1 \leq$ $M_{e x, k} \leq M_{A B}, k=1,2,3$. The value of $M_{e x, k}$ depends on how fast the kernel mode $\beta_{1}^{B A}(m)$ decays.

- given values of $\theta_{p}, \varphi_{q}$, and $r$ :

1. calculate the spectrum of the weighted spectrum: $t_{2}=\mathcal{F} \mathcal{S}\left[\hat{f}_{l}^{B} \beta_{1}^{B A}(l)\right]$.

2. apply fftshift to $\hat{f}_{m}^{A}$ and $\beta_{1}^{B A}(m)$, and let $t_{1}=\hat{f}_{m}^{A} \beta_{1}^{B A}(m)$.

3. in $\xi_{1}$ direction, calculate $t_{1}=\mathcal{S F}\left(t_{1}\right)$, select $N_{1}$ points from the first frequency node with a step $M_{e x, 1}$, apply the fftshift to the chosen data, and assign the final result to $t_{1}$.

4. repeat step 3 in the $\xi_{2}$ and $\xi_{3}$ directions.

5. let $t_{3}=t_{1} t_{2}$.

- sum up all $t_{3}$ for each set of $\theta_{p}, \varphi_{q}, r$, and the final result is the gain part of the collision operator $Q^{B A}$, that is, $Q^{B A+}$.

- the loss part of the collision operator $Q^{B A}$ is calculated in exactly the same way as that for the loss part of $Q^{A A}$, see Appendix B in Ref. [41].

In this new algorithm, memory usage does not depend on the mass ratio, since VDFs are discretized according Eqs. (26) and (27). The computational cost for the cross-collision operator $Q^{A B}$ is $O\left(\sqrt{m_{r}} M_{2} M^{2} N^{3} \log N\right)$. Since $\max \left(\xi_{l}^{A}\right)=\left(\pi / L_{A}\right)(N / 2), \max \left(\xi_{m}^{B}\right)=$ 

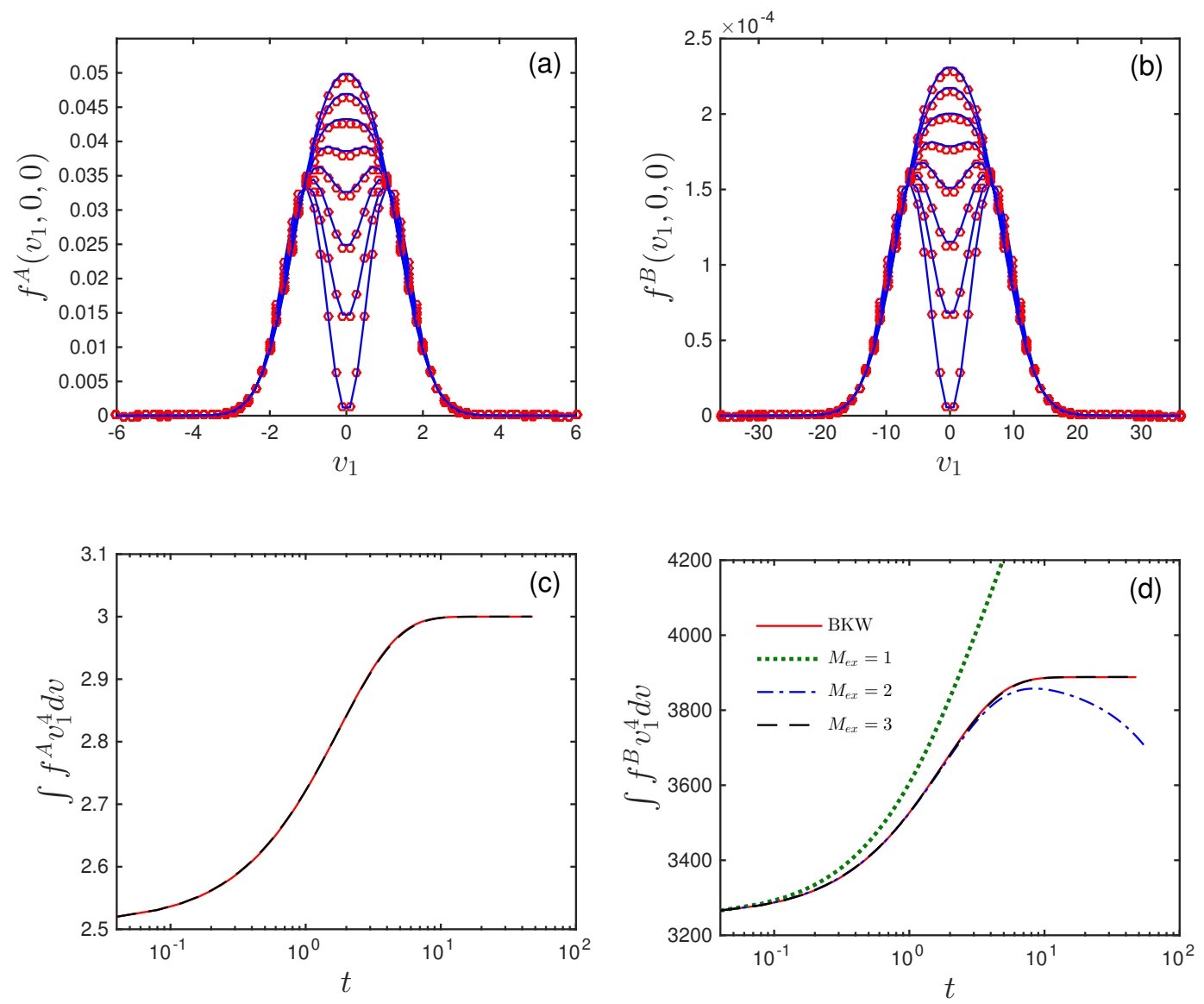

Figure 5: $(\mathrm{a}, \mathrm{b})$ The time evolution of VDFs in the spatially-homogeneous relaxation problem for pseudoMaxwell molecules. Solid lines in $(\mathrm{a}, \mathrm{b})$ represent exact BKW solutions of type I, while circles are from our FSM with $M_{e x}=2$. In each figure, from bottom to top (near $v_{1}=0$ ), the time is $(0,1,2, \cdots, 7) \times 0.25$. Parameters are the same as those used in the left column of Fig. 1, except here $m^{A} / m^{B}=36$. The velocity space is discretized by $64 \times 32 \times 32$ grids, while the frequency space is discretized by $32 \times 32 \times 32$ grids. We take $M=5, M_{2}=14$ in the calculation of $Q^{A B}$, while $M_{2}=32$ in the calculation of $Q^{B A}$. (c) The time evolution of fourth-order moments. Note that here we take $M_{e x, 1}=M_{e x, 2}=M_{e x, 3}=M_{e x}$.

$\left(\pi / L_{A} M_{A B}\right)(N / 2)$, and $\max \left(\rho_{r}\right)=L_{A} M_{A B}, \cos \left[\rho_{r}\left(a^{A B} \xi_{l}^{A}+b^{A B} \xi_{m}^{B}\right) \cdot e\right]$ in Eq. (18) oscillates $O(N)$ times, so $M_{2}$ should be $O(N)$, and the overall computational cost for $Q^{A B}$ is $O\left(\sqrt{m_{r}} M^{2} N^{4} \log N\right)$. In the calculation of $Q^{B A}$, since $\cos \left[\rho_{r}\left(a^{B A} \xi_{l}^{B}+b^{B A} \xi_{m}^{A}\right) \cdot e\right]$ oscillates $O\left(N M_{e x}\right)$ times, $M_{2}$ should be of the order of $N M_{e x}$. For $m_{r}$ up to 100, we have found that the case of $M_{e x}^{3} \sim m_{r}$ provides high accuracy, and the computational cost of $Q^{B A}$ is slightly higher than $Q^{A B}$. Compared to using the same velocity discretization for both VDFs, the new algorithm improves the computation efficiency significantly.

Note that it is possible to use a non-uniform discretization of the velocity space in Cartesian coordinates. The time penalty is negligible since the generation of a uniformly distributed spectrum from non-uniform velocity grids is of $O\left(N_{v}^{4} \log N_{v}\right)$, where $N_{v}$ is the number of velocity grid points in each velocity direction. This cost is much smaller than the overall computational cost. Our experience $[42,43]$ is that the number of frequency nodes 
can be much smaller than the number of velocity nodes, making FSM extremely efficient and accurate in capturing the discontinuities/fine structures in VDFs, which is useful in highly rarefied gas flows.

Figure 5 shows the relaxation-to-equilibrium process of pseudo-Maxwell molecules with a mass ratio $m_{r}=36$ using the new algorithm. The VDFs compare well with the exact BKW solutions when $M_{e x}=2$ (i.e. only $1 / 27$ of the frequency components of $\hat{f}^{A}$ is used in the calculation of $Q^{B A}$ ) when $t<10$. However, to make the fourth- and sixth-order moments of component $\mathrm{B}$ accurate over a long time, $M_{e x}$ is increased to 3.

\section{Numerical results for spatially-inhomogeneous problems}

It is convenient to use dimensionless variables. Here, we normalize the spatial location $x$ by the characteristic flow length $\ell$, the molecular velocity $v$ by the thermal speed of the A-component $v_{m}^{A}=\sqrt{2 k_{B} T_{0} / m^{A}}$ at the reference temperature $T_{0}$, the density $n$ by the reference molecular number density $n_{0}$, and the $\mathrm{VDF}$ by $n_{0} /\left(v_{m}^{A}\right)^{3}$. Then the $\mathrm{BE}(1)$ is recast to a dimensionless form, where the collision operator (2) with the collision kernel (6) is given by

$$
\begin{aligned}
Q^{\imath \jmath}\left(f^{\imath}, f^{\jmath}\right) & =\frac{1}{K n_{1}^{\imath \jmath}} \int_{\mathbb{R}^{3}} \int_{\mathbb{S}^{2}} \sin ^{\alpha^{\imath \jmath}+\gamma^{\imath \jmath}-1}\left(\frac{\theta}{2}\right) \cos ^{-\gamma^{\imath \jmath}}\left(\frac{\theta}{2}\right)|u|^{\alpha^{\imath \jmath}} f^{\jmath}\left({ }^{\prime} v_{*}^{\imath \jmath}\right) f^{\imath}\left({ }^{\prime} v^{\imath \jmath}\right) d \Omega d v_{*}-\nu^{\imath \jmath}\left(f^{\jmath}\right) f^{\imath}(v), \\
\nu^{\imath \jmath}\left(f^{\jmath}\right) & =\frac{1}{K n_{1}^{\imath \jmath}} \int_{\mathbb{R}^{3}} \int_{\mathbb{S}^{2}} \sin ^{\alpha^{\imath \jmath}+\gamma^{\imath \jmath}-1}\left(\frac{\theta}{2}\right) \cos ^{-\gamma^{\imath \jmath}}\left(\frac{\theta}{2}\right)|u|^{\alpha^{\imath \jmath}} f^{\jmath}\left(v_{*}\right) d \Omega d v_{*},
\end{aligned}
$$

where

$$
\begin{aligned}
K n_{1}^{\imath \jmath} & =\frac{64(2)^{\alpha^{\imath \jmath} / 2}}{5} \Gamma\left(\frac{\alpha^{\imath \jmath}+\gamma^{\imath \jmath}+3}{2}\right) \Gamma\left(2-\frac{\gamma^{\imath \jmath}}{2}\right) K n_{u n}^{\imath \jmath}, \\
\text { with } K n_{u n}^{\imath \jmath} & =\frac{\mu^{\imath \jmath}\left(T=T_{0}\right)}{n_{0} \ell} \sqrt{\frac{\pi}{2 m^{A} k_{B} T_{0}}},
\end{aligned}
$$

Note that here $\mu^{A B}$ should be understood as the shear viscosity when the collision kernel is given by Eq. (6) and $m^{A}=m^{B}$.

The molecular number density $n^{\imath}$ (normalized by $n_{0}$ ), flow velocity $U^{\imath}$ (normalized by $v_{m}^{A}$ ), and temperature $T^{\imath}$ (normalized by $T_{0}$ ) are defined as:

$$
n^{\imath}=\int f^{\imath} d v, \quad U^{\imath}=\frac{1}{n^{\imath}} \int v f^{\imath} d v, \quad T^{\imath}=\frac{2 m^{\imath}}{3 m^{A} n^{\imath}} \int\left|v-U^{\imath}\right|^{2} f^{\imath} d v .
$$

To obtain stationary solutions of the BE for spatially-inhomogeneous problems, the timedependent term is omitted. We then employ an iterative method to solve the BE: given the VDFs $f_{k}^{A}$ and $f_{k}^{B}$ at the $k$-th iteration step, their values at the $(k+1)$-th step are calculated by the following equations:

$$
\begin{aligned}
& {\left[\nu^{A A}\left(f_{k}^{A}\right)+\nu^{A B}\left(f_{k}^{B}\right)\right] f_{k+1}^{A}+v \cdot \frac{\partial f_{k+1}^{A}}{\partial x}=Q^{A A+}\left(f_{k}^{A}, f_{k}^{A}\right)+Q^{A B+}\left(f_{k}^{A}, f_{k}^{B}\right),} \\
& {\left[\nu^{B B}\left(f_{k}^{B}\right)+\nu^{B A}\left(f_{k}^{A}\right)\right] f_{k+1}^{B}+v \cdot \frac{\partial f_{k+1}^{B}}{\partial x}=Q^{B B+}\left(f_{k}^{B}, f_{k}^{B}\right)+Q^{B A+}\left(f_{k}^{B}, f_{k}^{A}\right),}
\end{aligned}
$$



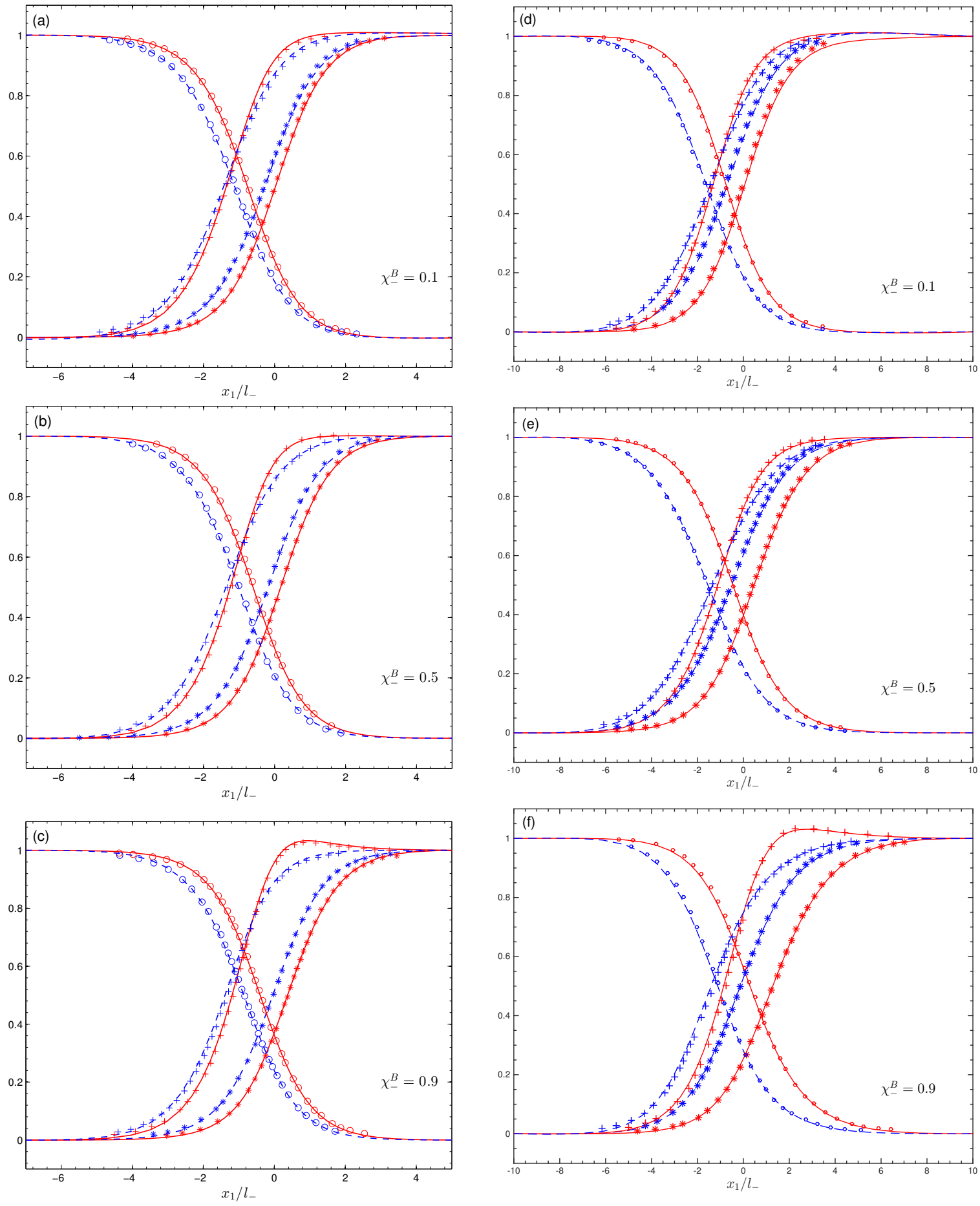

Figure 6: Normal shock wave profiles. (a-c) Molecular number densities (stars), flow velocities (circles), and temperatures (crosses) for an upstream Mach number of $3, m^{A} / m^{B}=2$, and $d_{m}^{B} / d_{m}^{A}=1$. Here $\chi_{-}^{B}$ is the concentration of the component $B$ at the upstream infinity. Lines are the numerical results of the FSM (solid: component A, dashed: component B), while symbols are the results of the numerical kernel method [50]. (d-f) As with (a-c), except that $m^{A} / m^{B}=4$ and the upstream Mach number is 2 . 
where the derivatives with respect to the spatial variable are approximated by second-order upwind finite differences. For the definition of $Q^{\imath \jmath+}$ and $\nu^{\imath \jmath}$, see Eqs. (2) and (3).

\subsection{Normal shock waves}

We compare our numerical solutions for normal shock waves with those obtained from the numerical kernel method [50]. The gas mixture is composed of hard-sphere molecules, with the diameters of components $\mathrm{A}$ and $\mathrm{B}$ being $d_{m}^{A}$ and $d_{m}^{B}$, respectively. The reference number density $n_{0}$ is chosen to be the molecular number density $n_{-}$at the upstream infinity, while the characteristic flow length $\ell$ is chosen as the mean free path $l_{-}$of the molecules of component $\mathrm{A}$ at the upstream infinity, i.e. $l_{-}=\left[\sqrt{2} \pi\left(d_{m}^{A}\right)^{2} n_{-}\right]^{-1}$. Therefore, in Eq. (28), $K n_{1}^{A A}=4 \sqrt{2} \pi, K n_{1}^{B B}=K n_{1}^{A A} /\left(d_{m}^{B} / d_{m}^{A}\right)^{2}$, and $K n_{1}^{A B}=K n_{1}^{B A}=4 K n_{1}^{A A} /\left(1+d_{m}^{B} / d_{m}^{A}\right)^{2}$.

Figure 6 shows the shock wave profiles in gases with different concentrations and Mach numbers. It can be seen that the results of the FSM compare well with those of the numerical kernel method.

\subsection{Heat transfer between two parallel plates}

Consider the heat transfer problem between two parallel plates. The gas mixture is composed of hard-sphere molecules, confined in the domain $0 \leq x_{1} \leq D$. The wall temperature at $x_{1}=0$ is $T_{I}$, and that at $x_{1}=D$ is $T_{I I}$. Maxwell's diffuse boundary condition is adopted at the two surfaces. For example, at $x_{1}=0$, VDFs for the reflected molecules are given as:

$$
\left.f^{\imath}\right|_{v_{1}>0}=n_{w}^{\imath}\left(\frac{m^{\imath}}{\pi T_{I} m^{A}}\right)^{3 / 2} \exp \left(-\frac{m^{\imath} v^{2}}{T_{I} m^{A}}\right),
$$

where $n_{w}^{\imath}=-2 \sqrt{\pi m^{\imath} / T_{I} m^{A}} \int_{v_{1}<0} v_{1} f^{\imath} d v$. The Knudsen number is

$$
K n=\frac{l_{0}}{D}
$$

where the mean-free path is $l_{0}=\left[\sqrt{2} \pi\left(d_{m}^{A}\right)^{2}\left(n_{a v}^{A}+n_{a v}^{B}\right)\right]^{-1}$.

In numerical simulations, we normalize the spatial coordinate $x_{1}$ by the plate separation $D$, and choose $n_{0}=n_{a v}^{A}+n_{a v}^{B}, T_{0}=T_{I}, K n_{1}^{A A}=4 \sqrt{2} \pi K n, K n_{1}^{B B}=K n_{1}^{A A} /\left(d_{m}^{B} / d_{m}^{A}\right)^{2}$, and $K n_{1}^{A B}=K n_{1}^{B A}=4 K n_{1}^{A A} /\left(1+d_{m}^{B} / d_{m}^{A}\right)^{2}$. Due to symmetry, the velocity region of the Acomponent $\left[-L_{A}, L_{A}\right] \times\left[-L_{A}, 0\right] \times\left[-L_{A}, 0\right]$ with $L_{A}=8.5$ is discretized by $64 \times 16 \times 16$ grid points, with $16 \times 16$ uniformly distributed points in the $v_{2}$ and $v_{3}$ directions, and $N_{v_{1}}=64$ nonuniform points in the $v_{1}$ direction:

$$
v_{1}^{A}=\frac{\left(-N_{v_{1}}+1,-N_{v_{1}}+3, \cdots, N_{v_{1}}-1\right)^{3}}{\left(N_{v_{1}}-1\right)^{3}} L_{A} .
$$

The number of frequency grid points is $32 \times 32 \times 32$. The discretization for the B-component is given by Eq. (27). To obtain the spectra of the VDFs, FFT is applied in the $v_{2}$ and $v_{3}$ directions, while in the $v_{2}$ direction Eq. (9) is discretized and the discrete Fourier transform is calculated by the trapezoidal rule. 


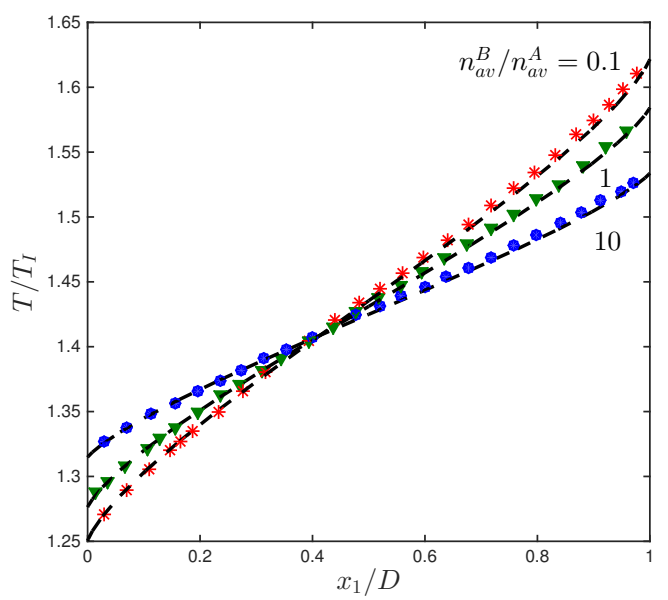

Figure 7: The average temperature $T=\left(n^{A} T^{A}+n^{B} T^{B}\right) /\left(n^{A}+n^{B}\right)$ in planar Fourier flow with $m^{A} / m^{B}=4$ and $K n=1$ for three number density ratios as labeled. Lines: FSM. Symbols: numerical kernel method [51].

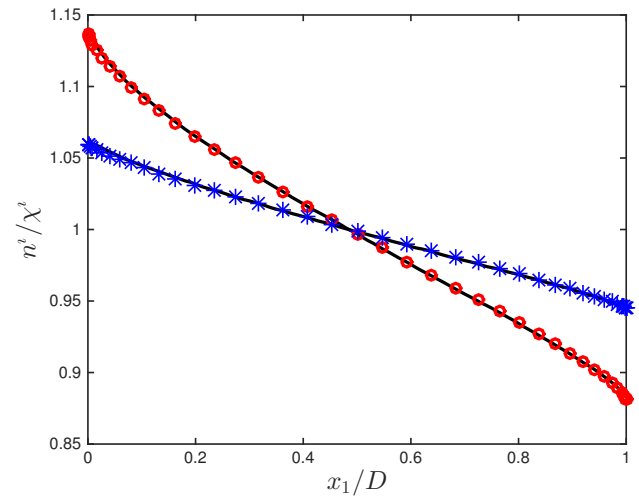

(a) Density

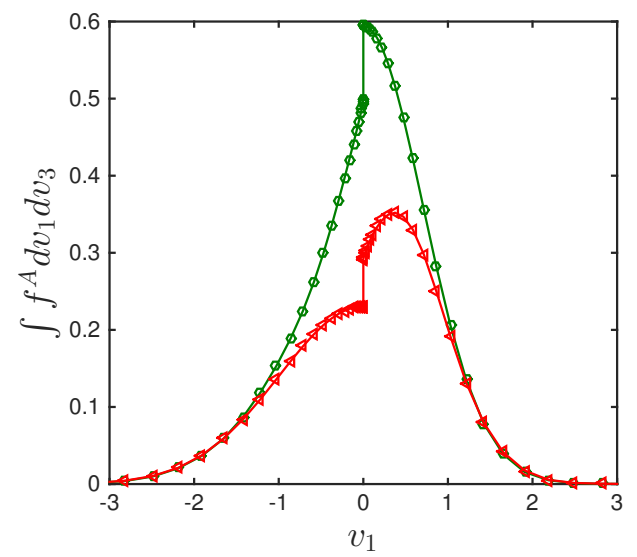

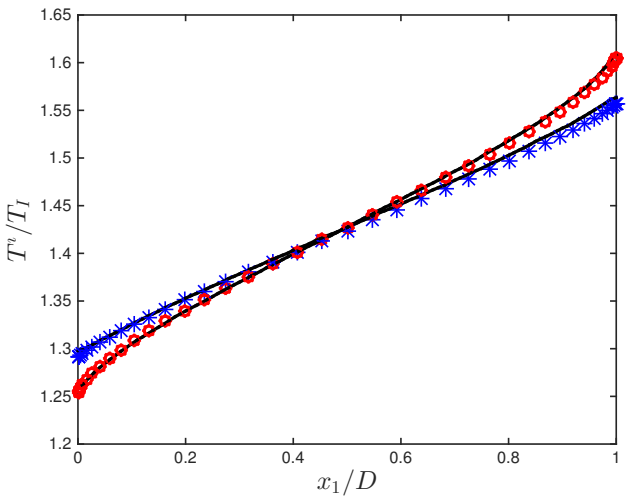

(b) Temperature

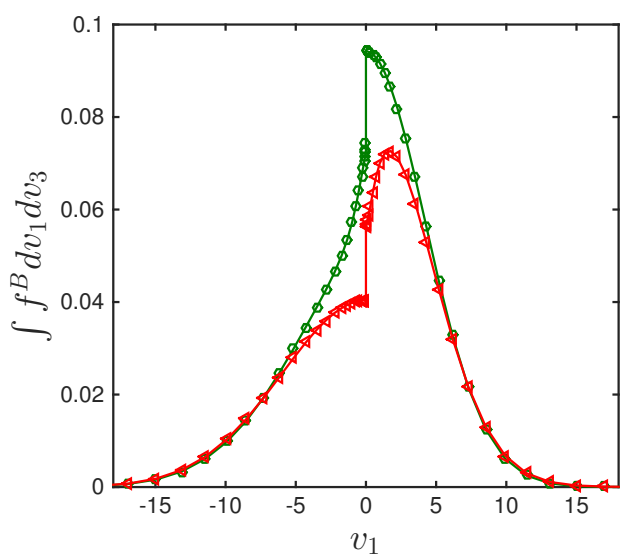

(c) Reduced VDFs

Figure 8: (a) Density and (b) temperature profiles with $m^{A} / m^{B}=36, n_{a v}^{B}=n_{a v}^{A}$, and $K n=1$. Circles (component A) and stars (component B) are the results from the FSM, while lines are from DSMC. (c) Reduced VDFs in the direction perpendicular to the wall. Circles (triangles): VDFs at $x_{1}=0\left(x_{1}=D\right)$. 
Figure 7 presents the temperature profiles at $K n=1, T_{I I} / T_{I}=2, d_{m}^{B} / d_{m}^{A}=0.5$, and for $n_{a v}^{B} / n_{a v}^{A}=0.1,1$, and 10 , where the mass ratio is 4 . The agreement with the results from the numerical kernel method [51] is quite satisfactory. We also investigate the same problem but for a molecular mass ratio as large as 36. Good agreement with DSMC results is shown in Fig. 8. The reduced VDFs are also shown, and the large discontinuities are demonstrably captured using the intensive non-uniform velocity grid. Note that in our numerical simulation we have 50 spatial grid points, and $M_{e x}=2$ is used in the approximation of $Q^{B A}$. Our Matlab code running on a PC with an Intel Xeon $3.3 \mathrm{GHz}$ CPU takes about 100 minutes to reduce the relative error in macroscopic quantities between two consecutive iteration steps to less than $10^{-5}$.
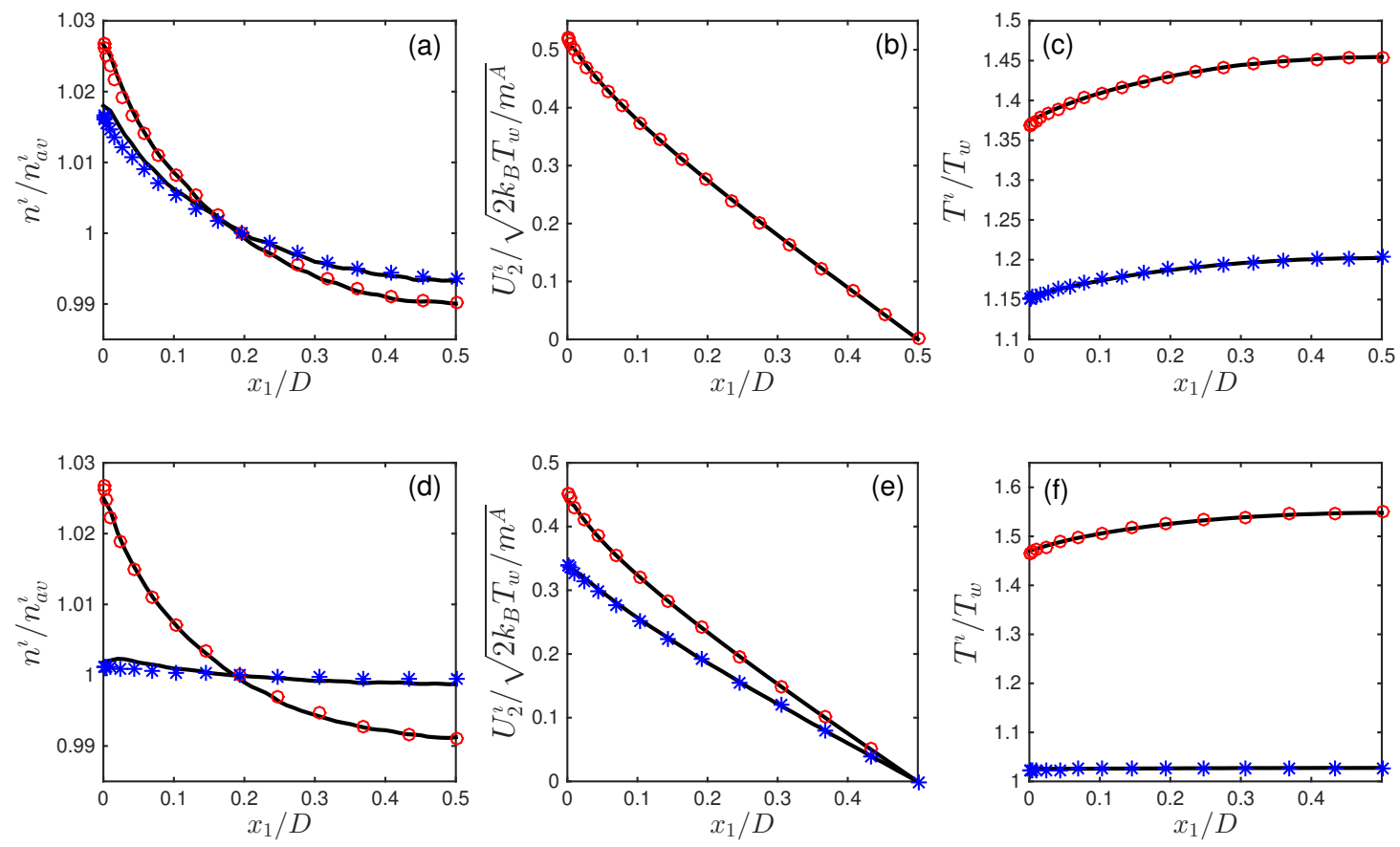

Figure 9: Molecular number density, velocity, and temperature profiles in planar Couette flow with $K n=1$ and $n_{a v}^{B}=n_{a v}^{A}$. (a-c) $m^{A} / m^{B}=4, d_{m}^{A}=d_{m}^{B}$. (d-f) $m^{A} / m^{B}=36, d_{m}^{A}=2 d_{m}^{B}$. Lines are the numerical results from DSMC, circles and stars are the results from our FSM for the components A and B, respectively. Note that in (b) the velocity profiles of the two components nearly coincide, so only that of the component A is shown. Because of the symmetry in this problem, only half of the spatial domain is shown.

\subsection{Planar Couette Flow}

Consider Couette flow between two parallel flat plates at temperature $T_{w}$. The plate at $x_{1}=0$ moves in the $x_{2}$ direction with a speed of $\sqrt{2 k_{B} T_{w} / m^{A}}$, while the plate at $x_{1}=D$ moves with the same speed but in the opposite direction. We consider hard-sphere molecules and Maxwell's diffuse boundary condition. At $x_{1}=0$, the boundary condition reads

$$
\left.f^{\imath}\right|_{v_{1}>0}=n_{w}^{\imath}\left(\frac{m^{\imath}}{\pi m^{A}}\right)^{3 / 2} \exp \left[-m^{\imath} \frac{v_{1}^{2}+\left(v_{2}-1\right)^{2}+v_{3}^{2}}{m^{A}}\right],
$$


(a)

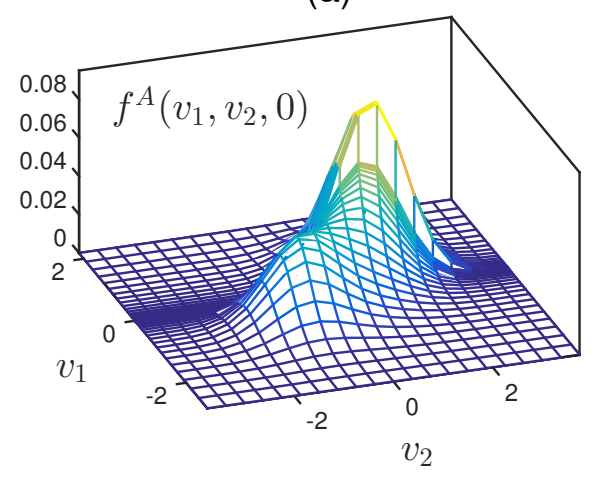

(c)

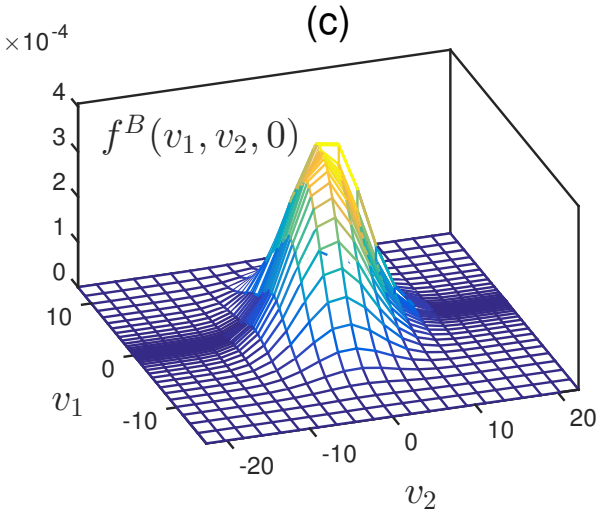

(b)

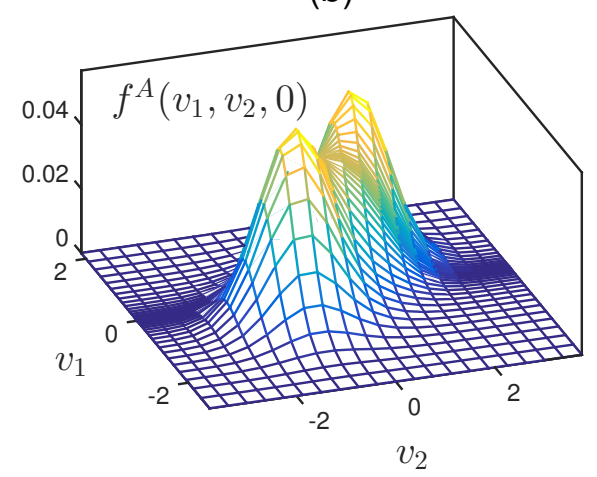

(d)

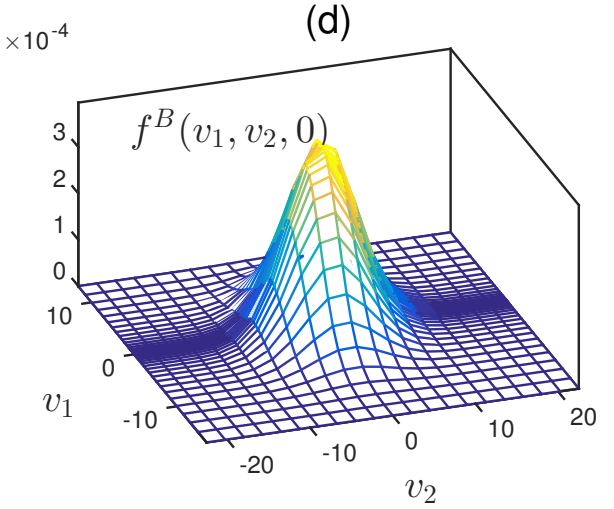

Figure 10: VDFs in planar Couette flow. The parameters are the same as in Fig. 9 (d-f). (a) and (c) VDFs at $x_{1}=0,(\mathrm{~b})$ and (d) VDFs at $x_{1}=D / 2$.

where $n_{w}^{\imath}=-2 \sqrt{\pi m^{\imath} / m^{A}} \int_{v_{1}<0} v_{1} f^{\imath} d v$.

The velocity region $[-6,6] \times[-6,6] \times[-6,0]$ has been discretized by 64 non-uniform grids in $v_{1}$ direction, and 32 and 16 uniform grids in the $v_{2}$ and $v_{3}$ directions, respectively. The number of frequency grid points is again $32 \times 32 \times 32$.

Figure 9 compares the molecular number density, flow velocity, and temperature profiles obtained using the FSM with those from DSMC, and excellent agreement is observed even when $M_{e x}=2$ for $m_{r}=36$. Note that for a mass ratio of 36 , the density $n^{B}$ and temperature $T^{B}$ only slightly deviate from their equilibrium values, since the wall speed is far smaller than the sound speed of the B-component. The density profile $n^{B}$ from the DSMC simulation is not well converged since DSMC finds it difficult to resolve small signals.

Figure 10 shows typical VDFs in the planar Couette flow. Since 64 non-uniform grids are used in the velocity direction normal to the wall (with most of the girds near $v_{1} \sim 0$ ), the discontinuities in the VDF near $v_{1} \sim 0$ are captured.

\subsection{Flow induced by periodic temperature variation}

Finally, we consider the flow of a gas mixture between two parallel plates that have a spatially-periodic temperature: the upper $\left(x_{2}=D\right)$ and lower plates $\left(x_{2}=0\right)$ have the 


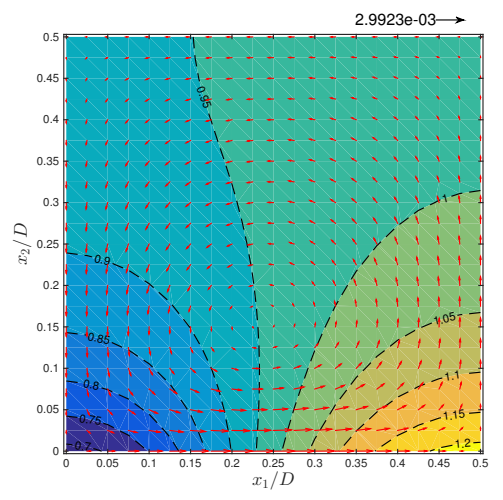

(a) $K n=1$, component A

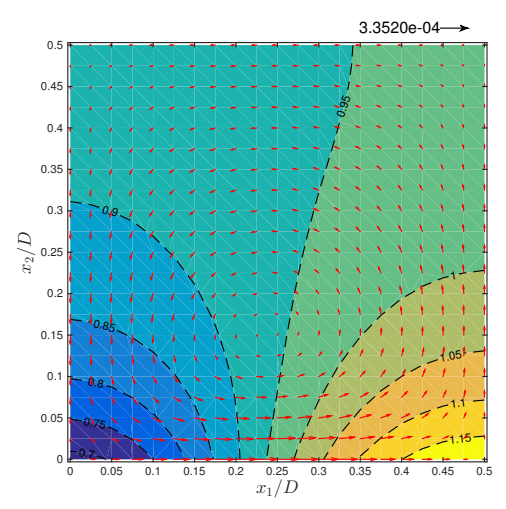

(d) $K n=10$, component A

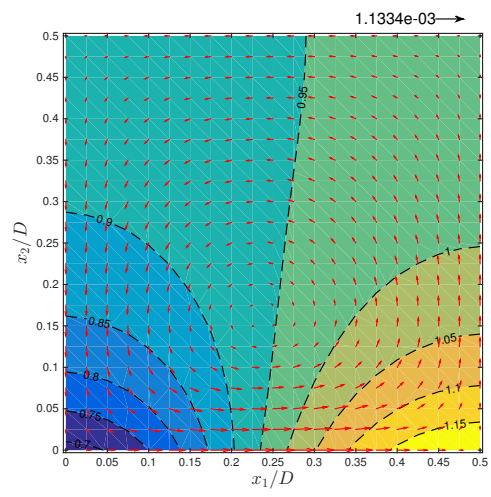

(b) $K n=1$, component B

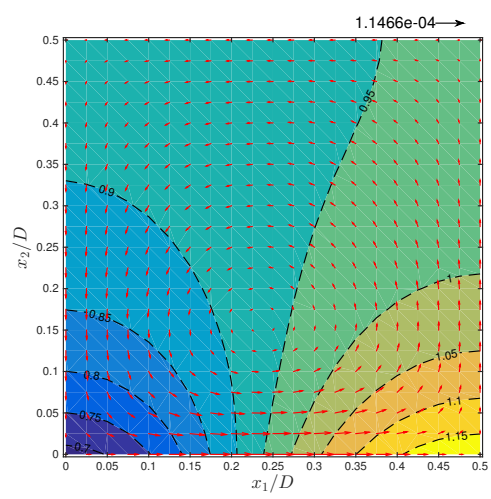

(e) $K n=10$, component $\mathrm{B}$

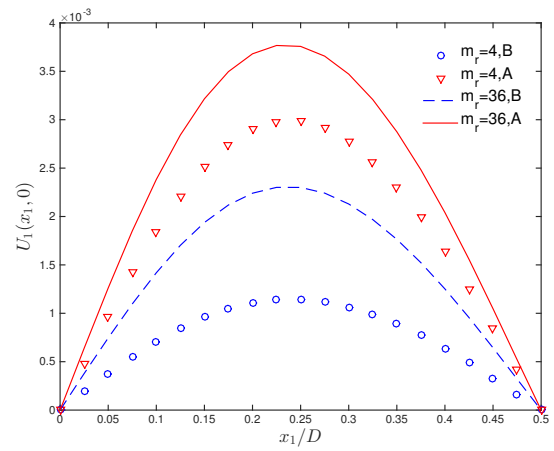

(c) $K n=1$

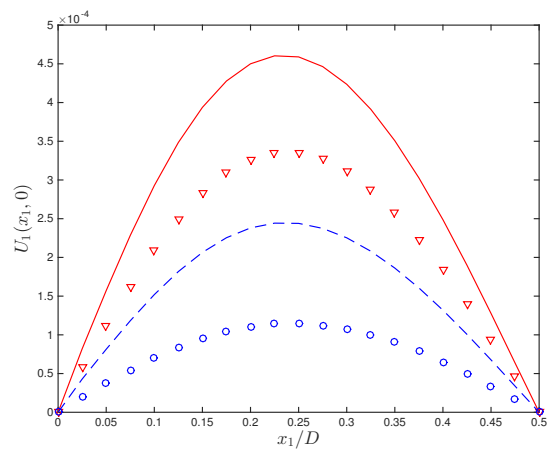

(f) $K n=10$

Figure 11: (a,b,d,e) Temperature contours and velocity vectors in the temperature-driven flow problem with $m^{A} / m^{B}=4$. The velocity is normalized by the maximum flow speed presented above each figure. (c,f) Comparison of the horizontal velocity at the wall between $m^{A} / m^{B}=4$ and 36 . Other parameters are $d_{m}^{A}=2 d_{m}^{B}$ and $n_{a v}^{B} / n_{a v}^{A}=1$

temperature $T_{0}\left(1-0.5 \cos 2 \pi x_{1} / D\right)$. Due to symmetry, the spatial domain $0 \leq x_{1}, x_{2} \leq D / 2$ is considered. The specular reflection boundary condition is chosen for the left, upper, and right boundaries, while the diffuse boundary condition is employed on the lower wall.

We choose a hard-sphere mixture of molecular mass ratio $\mathrm{m}^{A} / \mathrm{m}^{B}=4$, and the diameter of the lighter molecule is half that of the heavier one. Using the total mean density and the wall distance $D, K n$ is chosen to be 1 and 10 . The spatial domain is discretized by $21 \times 21$ equispaced points, while in the discretization of the velocity domain $\mathcal{D}_{L}=[-7.5,7.5)^{3}$, $168 \times 168 \times 16$ non-uniform grid points are used, see Eq. (34). The number of frequency components is $32 \times 32 \times 32$. Even with such a large number of velocity grid points, the FSM with $M=5, M_{2}=22$, and $M_{e x}=1$ takes only about 3 seconds to calculate the Boltzmann collision operators once at one spatial point. This is because FSM handles the collision in the frequency domain, and we only use $32 \times 32 \times 32$ frequency components.

The temperature and flow velocity is shown in Fig. 11. As $K n$ increases, the magnitude of the flow speed decreases. At the same $K n$, as the component $\mathrm{B}$ has a relatively large 

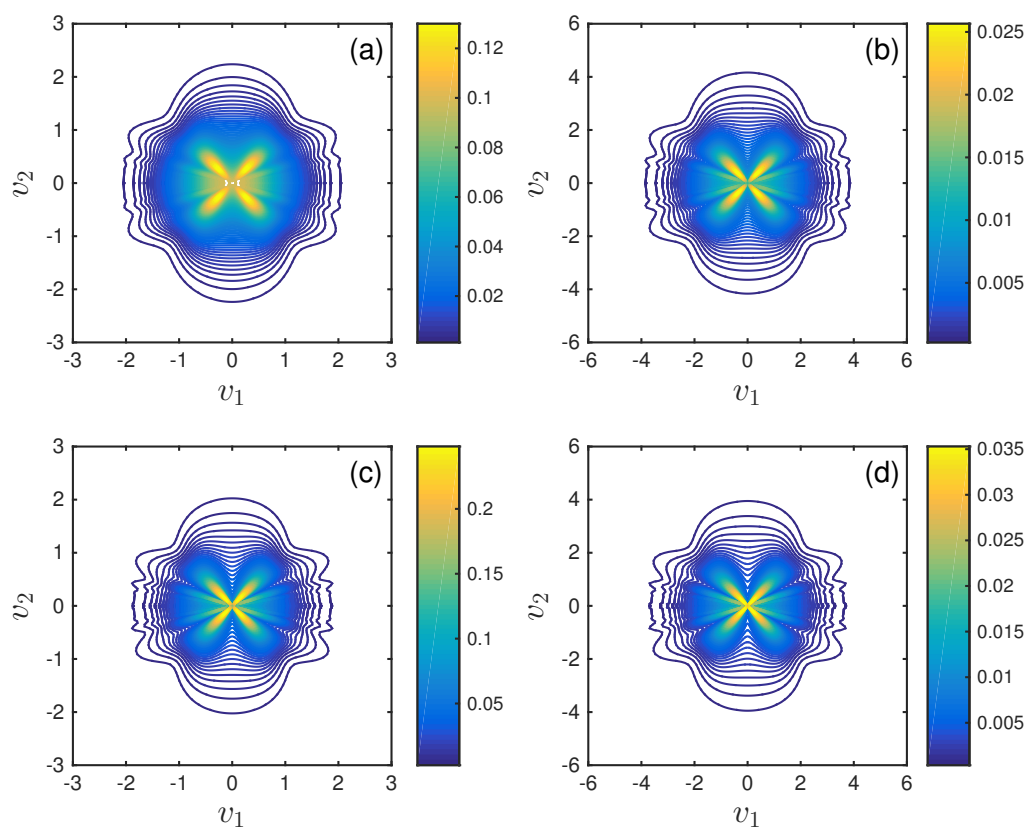

Figure 12: Contours of VDFs in the temperature-driven flow at $x_{1}=x_{2}=D / 2$. (a,c) $f^{A}\left(v_{1}, v_{2}, 0\right)$ and (b,d) $f^{B}\left(v_{1}, v_{2}, 0\right)$. The first row has $K n=1$ while the second row has $K n=10$.
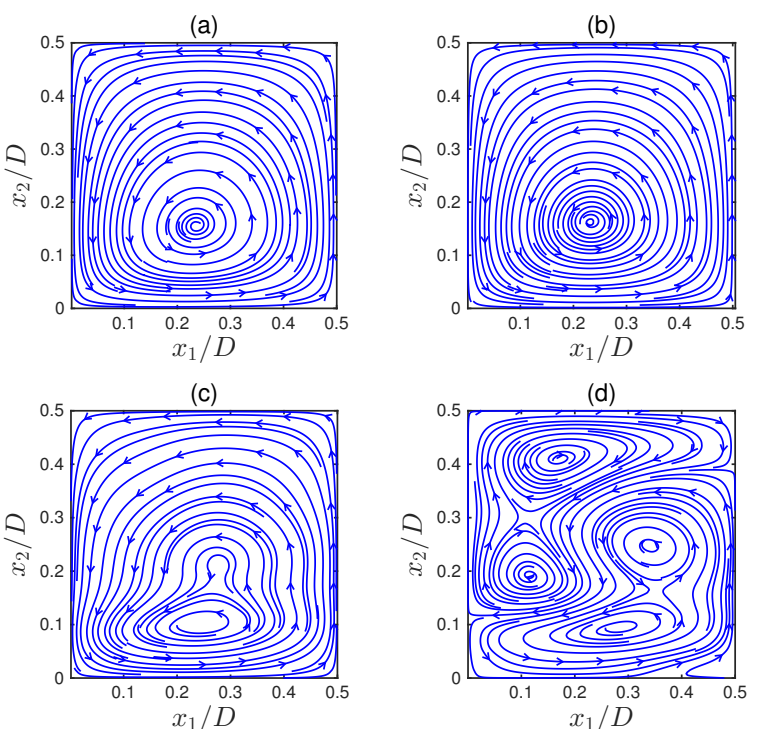

Figure 13: Velocity streamlines in the temperature driven flow with $K n=1$. (a,c) component A and (b,d) component B. The velocity grid points in the first row is $168 \times 168 \times 16$ (non-uniform), while that in the second row is $64 \times 64 \times 16$ (uniform). 
mean free path its overall flow speed is smaller than that of the component A. The case of $m^{A} / m^{B}=36$ is also simulated, and a comparison of the horizontal velocity at the wall is made. It is seen that the larger mass ratio case has larger flow speed at the wall, probably because the average molecular mass in the larger mass ratio case is smaller.

Figure 12 presents typical VDF profiles at $m^{A} / m^{B}=4$, where fine structures in the VDFs can be clearly seen. As $K n$ increases, the VDFs become more and more complicated, and hence more and more velocity grids are needed to capture these fine structures. To demonstrate the importance of using refined discretization in velocity space, we recalculate this problem using $64 \times 64 \times 16$ uniformly distributed velocity grid points at $K n=1$. The comparison of streamlines is presented in Fig. 13, which clearly shows that the coarser velocity grid cannot resolve the flow field. At $K n=10$, the streamlines from the $64 \times 64 \times 16$ uniform velocity grid are even worse. Other deterministic numerical methods, which deal with the binary collision in velocity space and have a higher computational cost, would have difficulty solving the flow field at large Knudsen numbers. Also, DSMC finds it extremely difficult to resolve the flow filed because of the small flow speed $\left(U \sim 10^{-4}\right)$.

\section{Conclusions}

We have developed a fast spectral method to solve the Boltzmann equation for binary mixtures of monatomic gases, with general forms of the collision kernel. The method employs Fourier-Galerkin discretization in velocity space, and handles binary collisions in the corresponding frequency space. The method has spectral accuracy, and can deal with mixtures with large molecular mass ratios at a computational cost of $O\left(\sqrt{m_{r}} M^{2} N^{4} \log N\right)$, which is much smaller than the conventional spectral method's cost of $O\left(m_{r}^{3} N^{6}\right)$ [57]. Numerical results have been presented for the spatially-homogeneous relaxation of pseudo-Maxwell molecules, and for spatially-inhomogeneous problems (such as normal shock waves, planar Fourier/Couette flows, and a two-dimensional temperature-driven flow) involving hardsphere gases with molecular mass ratios up to 36.

The fast spectral method can be extended straightforwardly to multispecies mixtures. Suppose we have a mixture of $n$ species; there are $n^{2}-n$ Boltzmann cross-collision operators. The storage of kernel modes is $O\left(n^{2} M^{2} N^{4}\right)$, which is tremendous. This problem, however, can be overcome if only $\psi(s)$ of Eq. (20) is stored, with a storage of $O\left(n^{2} N N_{s}\right)$, where $N_{s}$ is the discrete number of $s \in[0, \sqrt{3} \max (\xi)]$. The cost of this is that, in each time (iteration) step, $I^{\imath \jmath}\left(\xi_{l}, \xi_{m}, e_{\theta_{p}, \varphi_{q}}\right)$ is directly calculated, while $\psi\left(\sqrt{\left|\xi_{m}\right|^{2}-\left(\xi_{m} \cdot e_{\theta_{p}, \varphi_{q}}\right)^{2}}\right)$ in the kernel mode (19) is calculated by interpolation. This means that the computational cost for kernel modes is $O\left(n^{2} N^{4}\right)$, which is far less than the total computational cost for the collision operators of $O\left(\sqrt{m_{r}} n^{2} M^{2} N^{4} \log N\right)$.

Although we have only considered the hard-sphere and pseudo-Maxwell gases, the fast spectral method applies also to realistic intermolecular potentials. In this case, the computational cost for the self-collision operator increases from $O\left(M^{2} N^{3} \log N\right)$ to $O\left(M^{2} N^{4} \log N\right)$, which is less than that for cross-collision operators. Details of this will be given in a future paper. 
The fast spectral method turns out to be very good for highly rarefied gas flows, where a large number of velocity grids are needed to capture the discontinuities and fine structures in the velocity distribution functions, but the number of frequency components can be kept far smaller. For slightly rarefied flows, however, a large number of velocity discretizations is not necessary, but the spatial discretization needs to be fine. Since at small Knudsen number the moment method is accurate, in future work we shall consider coupling the fast spectral method to the recently-developed regularized moment equations [61].

\section{Acknowledgments}

The authors thank the reviewers of this paper for their valuable suggestions. This work is financially supported by the UK's Engineering and Physical Sciences Research Council (EPSRC) under grants EP/I036117/1 and EP/I011927/1.

\section{Appendix A. Transport coefficients}

To find the transport coefficients of the BE with the collision kernel (6), we first calculate the viscosity cross-section $\sigma_{\mu}^{\imath \jmath}$ :

$$
\sigma_{\mu}^{\imath \jmath}=2 \pi \int_{0}^{\pi} \frac{C^{\imath \jmath}}{|u|} \sin ^{3} \theta d \theta=16 \pi|u|^{\alpha^{\imath \jmath}-1} C_{0}^{\imath \jmath} \frac{\Gamma\left(\frac{\alpha^{\imath \jmath}+\gamma^{\imath \jmath}+3}{2}\right) \Gamma\left(2-\frac{\gamma^{\imath \jmath}}{2}\right)}{\Gamma\left(\frac{\alpha^{\imath \jmath}+7}{2}\right)},
$$

and the momentum transfer cross-section $\sigma_{M}^{\imath \jmath}$ :

$$
\sigma_{M}^{\imath \jmath}=2 \pi \int_{0}^{\pi} \frac{C^{\imath \jmath}}{|u|}(1-\cos \theta) \sin \theta d \theta=8 \pi|u|^{\alpha^{\imath \jmath}-1} C_{0}^{\imath \jmath} \frac{\Gamma\left(\frac{\alpha^{\imath \jmath}+\gamma^{\imath \jmath}+3}{2}\right) \Gamma\left(1-\frac{\gamma^{\imath \jmath}}{2}\right)}{\Gamma\left(\frac{\alpha^{\imath \jmath}+5}{2}\right)},
$$

where $\Gamma$ is the gamma function.

Then, according to the Chapman-Enskog expansion [3], the coefficient of the shear viscosity (when only the self-collision operator is considered) is given by

$$
\begin{aligned}
\mu^{\imath \imath} & =\frac{5 \sqrt{\pi m^{\imath} k_{B} T}}{8\left(m^{\imath} / 4 k_{B} T\right)^{4} \int_{0}^{\infty} u^{7} \sigma_{\mu}^{\imath \imath} \exp \left(-m^{\imath} u^{2} / 4 k_{B} T\right) d u} \\
& =\frac{5 \sqrt{\left(m^{\imath}\right)^{\alpha^{\imath \imath}} 4^{1-\alpha^{\imath \imath}} / \pi}}{64 C_{0}^{\imath \imath} \Gamma\left(\frac{\alpha^{\imath \imath}+\gamma^{2 \imath}+3}{2}\right) \Gamma\left(2-\frac{\gamma^{\imath 2}}{2}\right)}\left(k_{B} T\right)^{\omega^{\imath \imath}}
\end{aligned}
$$

while the diffusion coefficient is given by

$$
\begin{aligned}
& D^{\imath \jmath}=\frac{3 \sqrt{2 \pi k_{B} T / m_{r}^{\imath \jmath}}}{16\left(m_{r}^{\imath \jmath} / 2 k_{B} T\right)^{3} n \int_{0}^{\infty} u^{5} \sigma_{M}^{\imath \jmath} \exp \left(-m_{r}^{\imath \jmath} u^{2} / 2 k_{B} T\right) d u} \\
&=\frac{3 \sqrt{\left(m_{r}^{\imath \jmath}\right)^{\alpha^{\jmath \jmath}} 2^{2-\alpha^{\imath \jmath}} / \pi}}{64 C_{0}^{\imath \jmath} \Gamma\left(\frac{\alpha^{\imath \jmath}+\gamma^{\imath \jmath}+3}{2}\right) \Gamma\left(1-\frac{\gamma^{\imath \jmath}}{2}\right)} \frac{\left(k_{B} T\right)^{\omega^{\imath \jmath}}}{n m_{r}^{\imath \jmath}}, \\
& 24
\end{aligned}
$$


where

$$
\omega^{\imath \jmath}=1-\frac{\alpha^{\imath \jmath}}{2}
$$

$m_{r}^{\imath \jmath}=m^{\imath} m^{\jmath} /\left(m^{\imath}+m^{\jmath}\right)$ is the reduced mass, and $n$ is the total mass density: when $n=n^{A}+n^{B}$ when $\imath \neq \jmath$, and $n=n^{\imath}$ when $\imath=\jmath$.

It follows from Eqs. (A.3) and (A.5) that $\alpha^{i j}$ controls the temperature dependence of the shear viscosity and the diffusion, while $\gamma^{i j}$ controls the ratio between the diffusion and the shear viscosity. For self-collision operators, when the shear viscosity $\mu^{\imath \imath}$ is known (not only its value, but also its temperature dependence), we can determine $\alpha^{\imath \imath}$ from $\omega^{\imath \imath}$ and let $\gamma^{\imath \imath}$ be in the region $\left(-1-\alpha^{\imath \jmath}, 2\right)$ [41]. Then we determine $C_{0}^{\imath \imath}$ by Eq. (A.3).

For the cross-collision operators, when the coefficient of diffusion $D^{A B}$ is known (not only its value, but also its temperature dependence), we can determine $\alpha^{A B}$ from the value of $\omega^{A B}$, while the value of $\gamma^{A B}$ is determined by the ratio between the viscosity and momentum transfer cross-sections. Then, from Eq. (A.4) we determine $C_{0}^{A B}$.

For example, for a He-Ar mixture, since $\omega^{A B}=0.725[10]$, from Eq. (A.5) we choose $\alpha^{A B}=0.55$. As the ratio between the viscosity cross-section and the momentum transfer cross-section is $2 \times 1.64 /(1.64+2)$, from Eqs. (A.1) and (A.2) we choose $\gamma^{A B}=-0.50$.

\section{References}

[1] Y. Sone, Flows induced by temperature fields in a rarefied gas and their ghost effect on the behavior of a gas in the continuum limit, Annu. Rev. Fluid Mech. 32 (2000) 779-811.

[2] Y. Sone, Kinetic Theory and Fluid Dynamics, Birkhauser, 2002.

[3] S. Chapman, T. G. Cowling, The Mathematical Theory of Non-uniform Gases, Cambridge University, Cambridge, 1970.

[4] D. A. Lockerby, J. M. Reese, High-resolution Burnett simulations of micro Couette flow and heat transfer, J. Comput. Phys. 188 (2003) 333-347.

[5] L. S. Garcia-Colin, R. M. Velasco, F. J. Uribe, Beyond the Navier-Stokes equations: Burnett hydrodynamics. Phys. Rep. 465 (2008) 149-189.

[6] H. Grad, On the kinetic theory of rarefied gases, Commun. Pure Appl. Math. 2 (1949) 331-407.

[7] H. Struchtrup, Regularization of Grad's 13 moment equations: Derivation and linear analysis, Phys. Fluids, 15 (2003) 2668-2680.

[8] X. J. Gu, D. R. Emerson, A high-order moment approach for capturing non-equilibrium phenomena in the transition regime, J. Fluid Mech. 636 (2009) 177-216.

[9] H. Struchtrup, Macroscopic Transport Equations for Rarefied Gas Flows, Springer, 2005.

[10] G. A. Bird, Molecular Gas Dynamics and the Direct Simulation of Gas Flows, Clarendon Press, Oxford, 1994.

[11] N. G. Hadjiconstantinou, A. L. Garcia, M. Z. Bazant, G. He, Statistical error in particle simulations of hydrodynamic phenomena, J. Comput. Phys. 187 (2003) 274-297.

[12] J. Fan, C. Shen, Statistical simulation of low-speed rarefied gas flows, J. Comput. Phys, 167 (2001) 393-412.

[13] L. L. Baker, N. G. Hadjiconstantinou, Variance reduction for Monte Carlo solutions of the Boltzmann equation, Phys. Fluids, 17 (2005) 051703.

[14] T. M. M. Homolle, N. G. Hadjiconstantinou, A low-variance deviational simulation Monte Carlo for the Boltzmann equation, J. Comput. Phys. 226 (2007) 2341-2358.

[15] Q. H. Sun, I. D. Boyd, G. V. Boyd, A hybrid continuum/particle approach for micro-scale gas flows, AIP Conf. Proc. 663 (2003) 752-759. 
[16] T. E. Schwartzentruber, L. C. Scalabrin, I. D. Boyd, A modular particle-continuum numerical method for hypersonic non-equilibrium gas flows, J. Comput. Phys. 225 (2007) 1159-1174.

[17] J. M. Burt, I. D. Boyd, A hybrid particle approach for continuum and rarefied flow simulation, J. Comput. Phys. 228 (2009) 460-475.

[18] A. Alaia, G. Puppo, A hybrid method for hydrodynamic-kinetic flow Part I: A particle-grid method for reducing stochastic noise in kinetic regimes, J. Comput. Phys. 230 (2011) 5660-5683.

[19] A. Alaia, G. Puppo, A hybrid method for hydrodynamic-kinetic flow Part II: Coupling of hydrodynamic and kinetic models, J. Comput. Phys. 231 (2012) 5217-5242.

[20] P. Degond, G. Dimarco, Fluid simulations with localized Boltzmann upscaling by direct simulation Monte-Carlo, J. Comput. Phys. 231 (2012) 2414-2437.

[21] S. Y. Docherty, M. K. Borg, D. A. Lockerby, J. M. Reese, Multiscale simulation of heat transfer in a rarefied gas, Int. J. Heat Fluid, 50 (2014) 114-125.

[22] L. Pareschi, G. Russo, Asymptotic preserving Monte Carlo methods for the Boltzmann equation, Transp. Theo. Stat. Phys. 29 (2000) 415-430.

[23] L. Pareschi, G. Russo, Time relaxed Monte Carlo methods for the Boltzmann equation, SIAM J. Sci. Comput. 23 (2001) 1253-1273.

[24] S. Tiwari, A. Klar, S. Hardt, A particle-particle hybrid method for kinetic and continuum equations, J. Comput. Phys. 228 (2009) 7109-7124.

[25] M. H. Gorji, P. Jenny, Fokker-Planck-DSMC algorithm for simulations of rarefied gas flows, J. Comput. Phys. 287 (2015) 110-129.

[26] M. K. Borg, D. A. Lockerby, J. M. Reese, A multiscale method for micro/nano flows of high aspect ratio, J. Comput. Phys. 233 (2013) 400-413.

[27] A. Patronis, D. A. Lockerby, M. K. Borg, J. M. Reese, Hybrid continuum-molecular modelling of multiscale internal gas flows, J. Comput. Phys. 255 (2013) 558-571.

[28] A. Patronis, D. A. Lockerby, Multiscale simulation of non-isothermal microchannel gas flows, J. Comput. Phys. 270 (2014) 532-543.

[29] Y. Sone, T. Ohwada, K. Aoki, Temperature jump and Knudsen layer in a rarefied gas over a plane wall: Numerical analysis of the linearized Boltzmann equation for hard-sphere molecules, Phys. Fluids A 1 (1989) 363-370.

[30] T. Ohwada, Structure of normal shock waves: Direct numerical analysis of the Boltzmann equation for hard-sphere molecules, Phys. Fluids 5 (1993) 217-234.

[31] V. V. Aristov, Direct Methods for Solving the Boltzmann Equation and Study of Nonequilibrium Flows, Kluwer Academic Publishers, 2001.

[32] F. G. Tcheremissine, Direct numerical solution of the Boltzmann equation, AIP Conf. Proc. 762 (2005) 677-685.

[33] L. Pareschi, B. Perthame, A Fourier spectral method for homogeneous Boltzmann equation, Transport Theory Statist. Phys. 25 (1996) 369-382.

[34] L. Pareschi, G. Russo, Numerical solution of the Boltzmann equation I: Spectrally accurate approximation of the collision operator, SIAM J. Numer. Anal. 37 (2000) 1217-1245.

[35] C. Mouhot, L. Pareschi, Fast algorithms for computing the Boltzmann collision operator, Math. Comput. 75 (2006) 1833-1852.

[36] F. Filbet, C. Mouhot, L. Pareschi, Solving the Boltzmann equation in $N \log _{2} N$, SIAM J. Sci. Comput. 28 (2006) 1029-1053.

[37] I. M. Gamba, S. H. Tharkabhushanam, Spectral-Lagrangian methods for collisional models of nonequilibrium statistical states, J. Comput. Phys. 228 (2009) 2012-2036.

[38] A. Majorana, A numerical model of the Boltzmann equation related to the discontinuous Galerkin method, Kinet. Relat. Models, 4 (2011) 139-151.

[39] A. Alekseenko, E. Josyula, Deterministic solution of the spatially homogeneous Boltzmann equation using discontinuous Galerkin discretizations in the velocity space, J. Comput. Phys. 272 (2014) 170188.

[40] G. P. Ghiroldi, L. Gibelli, A direct method for the Boltzmann equation based on a pseudo-spectral 
velocity space discretization, J. Comput. Phys. 258 (2014) 568-584.

[41] L. Wu, C. White, T. J. Scanlon, J. M. Reese, Y. Zhang, Deterministic numerical solutions of the Boltzmann equation using the fast spectral method, J. Comput. Phys. 250 (2013) 27-52.

[42] L. Wu, J. M. Reese, Y. Zhang, Solving the Boltzmann equation deterministically by the fast spectral method: application to gas microflows, J. Fluid Mech. 746 (2014) 53-84.

[43] L. Wu, J. M. Reese, Y. Zhang, Oscillatory rarefied gas flow inside rectangular cavities, J. Fluid Mech. 748 (2014) 350-367.

[44] V. I. Kolobov, R. R. Arslanbekov, V. V. Aristov, A. A. Frolova, S. A. Zabelok, Unified solver for rarefied and continuum flows with adaptive mesh and algorithm refinement, J. Comput. Phys. 223 (2007) 589-608.

[45] T. Ohwada, Y. Sone, K Aoki, Numerical analysis of the Poiseuille and thermal transpiration flows between two parallel plates on the basis of the Boltzmann equation for hard sphere molecules, Phys. Fluids, 1 (1989) 2042-2049.

[46] Yu. A. Anikin, O. I. Dodulad, Yu. Yu. Kloss, F. G. Tcheremissine, Method of calculating the collision integral and solution of the Boltzmann kinetic equation for simple gases, gas mixtures and gases with rotational degrees of freedom, Int. J. Comput. Math. (2014) 1-14. http://dx.doi.org/10.1080/00207160.2014.909033.

[47] F. Filbet, On deterministic approximation of the Boltzmann equation in a bounded domain, Multiscale Model. Simul. 10 (2012) 792-817.

[48] L. Wu, C. While, T. J. Scanlon, J. M. Reese, Y. Zhang, Coherent Rayleigh-Brillouin scattering: Influence of the intermolecular potential, AIP Conf. Proc. 1628 (2014) 648-652.

[49] L. Wu, C. While, T. J. Scanlon, J. M. Reese, Y. Zhang, A kinetic model of the Boltzmann equation for non-vibrating polyatomic gases, J. Fluid Mech. 763 (2015) 24-50.

[50] S. Kosuge, K. Aoki, S. Takata, Shock-wave structure for a binary gas mixture: finite-difference analysis of the Boltzmann equation for hard-sphere molecules, Eur. J. Mech. B/Fluids, 20 (2001) 87-126.

[51] S. Kosuge, K. Aoki, S. Takata, Heat transfer in a gas mixture between two parallel plates: Finitedifference analysis of the Boltzmann equation, AIP Conf. Proc. 585 (2000) 289-296.

[52] S. Takata, S. Yasuda, S. Kosuge, K. Aoki, Numerical analysis of thermal-slip and diffusion-slip flows of a binary mixture of hard-sphere molecular gases, Phys. Fluids 15 (2003) 3745-3766.

[53] R. D. M. Garcia, C. E. Siewert, The viscous-slip, diffusion-slip, and thermal-creep problems for a binary mixture of rigid spheres described by the linearized Boltzmann equation, Eur. J. Mech. B/Fluids, 26 (2007) 749778 .

[54] R. D. M. Garcia, C. E. Siewert, Couette flow of a binary mixture of rigid-sphere gases described by the linearized Boltzmann equation, Eur. J. Mech. B/Fluids, 27 (2008) 823-836.

[55] R. D. M. Garcia, C. E. Siewert, The linearized Boltzmann equation with Cercignani-Lampis boundary conditions: Basic flow problems in a plane channel, Eur. J. Mech. B/Fluids, 28 (2009) 387396.

[56] O. I. Dodulad, F. G. Tcheremissine, Multipoint conservative projection method for computing the Boltzmann collision integral for gas mixtures, AIP Conf. Proc. 1501 (2012) 302-309.

[57] A. Munafòa, J. R. Haack, I. M. Gamba, T. E. Magin, A spectral-Lagrangian Boltzmann solver for a multi-energy level gas, J. Comput. Phys. 264 (2014) 152-176.

[58] J. Hu, L. Ying, A fast spectral algorithm for the quantum Boltzmann collision operator, Commun. Math. Sci. 10 (2012) 989-999.

[59] M. Krook, T. T. Wu, Exact solution of Boltzmann equation for multicomponent systems, Phys. Rev. Lett. 38 (1977) 991-993.

[60] Y. N. Lu, Y. T. Xiong, Solutions of the Boltzmann equation for the spatially homogeneous mixture of Maxwellian gases, Commun. Theor. Phys. 6 (1986) 17-28.

[61] V. K. Gupta, M. Torrilhon, Higher order moment equations for rarefied gas mixtures, Proc. R. Soc. A 471 (2015) 20140754. 